\title{
TEICHMÜLLER SPACE FOR ITERATED FUNCTION SYSTEMS
}

\author{
MARTIAL R. HILLE AND NINA SNIGIREVA
}

\begin{abstract}
In this paper we investigate families of iterated function systems (IFS) and conformal iterated function systems (CIFS) from a deformation point of view. Namely, we introduce the notion of Teichmüller space for finitely and infinitely generated (C)IFS and study its topological and metric properties. Firstly, we completely classify its boundary. In particular, we prove that this boundary essentially consists of inhomogeneous systems. Secondly, we equip Teichmüller space for (C)IFS with different metrics, an Euclidean, a hyperbolic, and a $\lambda$-metric. We then study continuity of the Hausdorff dimension function and the pressure function with respect to these metrics. We also show that the hyperbolic metric and the $\lambda$-metric induce topologies stronger than the non-metrizable $\lambda$-topology introduced by Roy and Urbanski and, therefore, provide an alternative to the $\lambda$-topology in the study of continuity of the Hausdorff dimension function and the pressure function. Finally, we investigate continuity properties of various limit sets associated with infinitely generated (C)IFS with respect to our metrics.
\end{abstract}

\section{INTRODUCTION}

Iterated function systems (IFS), introduced by Hutchinson Hut81 in 1981 (the terminology is due to Barnsley, see e.g. Bar93) and their generalisations conformal iterated function systems (CIFS) have been studied thoroughly in the last 25 years. Recently, there has been an increased interest to study families of (C)IFS instead of single (C)IFS (see e.g. [RU05, BBG, KS08]). In particular, continuity of functions such as the Hausdorff dimension function or the pressure function on the space of (C)IFS have been investigated (see e.g. [RU05, RSU09]). For this reason the space of infinitely generated (C)IFS (which satisfy certain separation conditions) has been equipped with a topology, called the $\lambda$-topology, which turned out not to be metrizable. To the best of our knowledge, a systematic approach to study families of (C)IFS following Teichmüller theory-ideas has not yet been introduced, with exception of the paper [KS08 where the moduli space of CIFS has been introduced. We also note that the work of McMullen and Sullivan [MS98] and Pinto et all (see e.g. PRF08 and references therein) on Teichmüller theory for certain dynamical systems differ considerably from our approach. In particular, the language used in the forementioned works is very similar to the language of Teichmüller theory for Riemann surfaces. In this paper, we translate the ideas of Teichmüller theory into the language of iterated function systems and exclusively use the parameter space of (C)IFS.

Received by the editors November 29, 2011.

2010 Mathematics Subject Classification. Primary 37F45, 37F35, 37F40, 28A80.

Key words and phrases. Iterated function systems, inhomogeneous iterated function systems, conformal iterated function systems, Teichmüller space, Hausdorff dimension, $\lambda$-topology. 
It is well known that the Teichmüller space for a Riemann surface $X$ is the space of (certain equivalent classes of) metrics on $X$, and is strongly related to the socalled parameter space. An important part of Teichmüller theory is the study of the boundary of Teichmüller space. In this paper we introduce Teichmüller space for IFS in any dimension and for CIFS in dimension $d \geq 3$ with a given number of finite or countably infinite generators and satisfying certain separation conditions. This will be done using the parameter space of these iterated function systems. We then give a full classification of the boundary of Teichmüller space (see Theorems 3.4 and 4.1). It turns out that essentially this boundary consists of inhomogeneous systems. For finitely generated IFS, such inhomogeneous systems were introduced in BD85, Bar89, Bar93, Bar06] and investigated further in OS07, OS08a, OS08b]. Finitely generated inhomogeneous IFS originate from the study of condensed Julia sets which arise naturally as deformations of Julia sets for rational maps BGH85. The fact that the boundary essentially consists of inhomogeneous systems is in agreement with these earlier studies of deformations of Julia sets and clarifies how inhomogeneous systems arise naturally in the framework of Teichmüller theory for (C)IFS. To classify the boundary of Teichmüller space for CIFS, we extend the notion of inhomogeneous systems to finitely and infinitely generated CIFS. We also include a discussion of the inhomogeneous sets associated to inhomogeneous CIFS. In the sequel in preparation $\mathrm{HSb}$ ] we plan to extend these notions to (conformal) graph directed Markov systems.

It is natural to metricize Teichmüller space for (C)IFS. We will do this by introducing appropriate metrics on Teichmüller space for (C)IFS. One of these metrics arises naturally from a hyperbolic metric and the other one from an Euclidean metric. We also introduce the $\lambda$-metric, convergence with respect to which implies convergence in the $\lambda$-topology. We then show that the essential boundary of Teichmüller space for finitely generated IFS is exactly given by the completion with respect to the Euclidean metric (see Corollary 3.12). Furthermore, we show that a similar statement does not hold for CIFS. The hyperbolic metric allows us to distinguish different parts of the boundary of Teichmüller space for (C)IFS since we prove that, in contrast to the rest of the essential boundary, the part of the boundary consisting of inhomogeneous systems is infinitely far away from the interior with respect to the hyperbolic metric (see Propositions 3.11 and 4.3 ).

Finally, we study continuity of the Hausdorff dimension function and the pressure function with respect to the metrics introduced. In particular, we find that the Hausdorff dimension function for finitely generated IFS extends continuously with respect to the Euclidean metric to the essential boundary of Teichmüller space for IFS (see Theorem 6.1). Moreover, we show that the hyperbolic metric on Teichmüller space for infinitely generated IFS and the $\lambda$-metric on Teichmüller space for infinitely generated (C)IFS have indeed some very nice properties related to the $\lambda$-topology. More precisely, we prove that convergence with respect to the hyperbolic metric for IFS and the $\lambda$-metric for (C)IFS implies convergence with respect to the $\lambda$-topology (see Theorem 5.1 and Proposition 7.4). From this follows the continuity of the Hausdorff dimension function and the pressure function with respect to the hyperbolic metric for IFS and the $\lambda$-metric for (C)IFS (see Corollary 7.6). We conclude the paper by studying continuity properties of various limit sets associated with infinitely generated (C)IFS with respect to the metrics introduced (see Theorems 9.1, 9.2 and 9.3). These metrics induce very strong topologies in a 
sense that most of the maps we investigate in this paper are shown to be continuous with respect to our metrics and the maps which are shown to be discontinuous in general can only be made continuous with respect to the discrete metric. This shows that our metrics are good for studying deformations of (C)IFS.

\section{Preliminaries}

2.1. Iterated function systems. Let $I \subset \mathbb{N}$ be a finite index set and let $\mathbf{S}=$ $\left\{S_{i}\right\}_{i \in I}$ be a set of contracting similarities on $\mathbb{R}^{d}$. The set $\mathbf{S}$ is called an Iterated Function System (IFS) [Bar89, Bar93. The notion of an IFS extends readily to the notion of an Inhomogeneous Iterated Function System (IIFS) defined by $\mathbf{S}_{C}=$ $(\mathbf{S}, C)$ where $C$ is a compact subset of $\mathbb{R}^{d}$ [BD85, Bar89, Bar93, Bar06].

Remark 2.1. In BD85, Bar89, Bar93, Bar06] the system $\mathbf{S}_{C}$ is called an Iterated Function System with condensation and the set $C$ is called the condensation set.

In Hut81] it was shown that there is a unique non-empty compact set $L(\mathbf{S}) \subset \mathbb{R}^{d}$, called the self-similar set associated with the IFS $\mathbf{S}$ or the limit set generated by the IFS S, such that

$$
L(\mathbf{S})=\bigcup_{i \in I} S_{i}(L(\mathbf{S}))
$$

Similarly, in BD85 it was shown that there is a unique non-empty compact set $L\left(\mathbf{S}_{C}\right) \subset \mathbb{R}^{d}$, called the self-similar set with condensation or the inhomogeneous self-similar set associated with the IIFS $\mathbf{S}_{C}$ (see OS07, OS08a, OS08b]) or the inhomogeneous limit set generated by the IIFS $\mathbf{S}_{C}$, such that

$$
L\left(\mathbf{S}_{C}\right)=\bigcup_{i \in I} S_{i}\left(L\left(\mathbf{S}_{C}\right)\right) \cup C .
$$

To study the geometry of the limit set $L(\mathbf{S})$ it is often useful to impose various natural separation conditions on $\mathbf{S}$. We say that $\mathbf{S}$ satisfies the Strong Separation Condition (SSC) if $S_{i}(L(\mathbf{S})) \cap S_{j}(L(\mathbf{S}))=\emptyset$ for all $i, j \in I$ with $i \neq j$. We say that $\mathbf{S}$ satisfies the Open Set Condition (OSC) if there exists an open non-empty and bounded subset $U$ of $\mathbb{R}^{d}$ such that $\bigcup_{i \in I} S_{i}(U) \subseteq U$ and $S_{i}(U) \cap S_{j}(U)=\emptyset$ for all $i, j \in I$ with $i \neq j$.

Similarly, to study the geometry of the inhomogeneous limit set $L\left(\mathbf{S}_{C}\right)$ it is useful to introduce the inhomogeneous version of SSC. We say that $L\left(\mathbf{S}_{C}\right)$ satisfies the Inhomogeneous Strong Separation Condition (ISSC) if the sets $\left(S_{i}\left(L\left(\mathbf{S}_{C}\right)\right)\right)_{i \in I}, C$ are pairwise disjoint [S07. We say that $\mathbf{S}$ satisfies the Inhomogeneous Open Set Condition (IOSC) if there exists an open non-empty and bounded subset $U$ of $\mathbb{R}^{d}$ such that $\bigcup_{i \in I} S_{i}(U) \subseteq U$ and the sets $\left\{S_{i}(U)\right\}_{i \in I}, C$ are pairwise disjoint.

2.2. Conformal iterated function systems. An IFS $\mathbf{S}=\left\{S_{i}\right\}_{i \in I}$ is called conformal (CIFS) acting on the phase space $X$ if the following conditions are satisfied:

(1) $X$ is a compact connected subset of $\mathbb{R}^{d}$ such that $X=\overline{\int_{\mathbb{R}^{\mathrm{d}}}(X)}$. Here, $\int_{\mathbb{R}^{\mathrm{d}}}(X)$ denotes the interior of $X$ and $\overline{\int_{\mathbb{R}^{\mathrm{d}}}(X)}$ denotes the closure of $\int_{\mathbb{R}^{\mathrm{d}}}(X)$.

(2) $\mathbf{S}$ satisfies the OSC with $U=\int_{\mathbb{R}^{\mathrm{d}}}(X)$.

(3) There exists an open connected set $V$ with $X \subset V \subset \mathbb{R}^{d}$ such that all maps $S_{i}, i \in I$, extend to $C^{1}$ conformal diffeomorphisms of $V$ into $V$. 
(4) There exists $\gamma, l>0, \gamma<\frac{\pi}{2}$ such that for every $x \in X$ there exists an open cone $\operatorname{Con}(x, \gamma, l) \subset \int_{\mathbb{R}^{\mathrm{d}}}(X)$ with vertex $x$, central angle of Lebesgue measure $\gamma$, and altitude $l$.

(5) There are two constants $L \geq 1$ and $\alpha>0$ such that

$$
|| S_{i}^{\prime}(y)|-| S_{i}^{\prime}(x)|| \leq \frac{L}{\left\|\left(S_{i}^{\prime}\right)^{-1}\right\|_{X}}\|y-x\|^{\alpha}
$$

for all $x, y \in X$ and all $i \in I$, where $\|\cdot\|_{X}$ is the supremum norm taken over $X$.

As in the case of IFS, the notion of CIFS extends readily to the notion of an Inhomogeneous Conformal Iterated Function System (ICIFS) defined by $\mathbf{S}_{C}=$ $(\mathbf{S}, C)$ where $C$ is a compact subset of $X$ and Condition (2) reads as: $\mathbf{S}_{C}$ satisfies IOSC with $U=\int_{\mathbb{R}^{\mathrm{d}}}(X)$.

It is obviously possible that $S_{i}(X) \cap S_{j}(X)=\emptyset$ for all $i, j \in I$ with $i \neq j$. In this case we will say that $\mathbf{S}$ satisfies SSC. Similarly we say that $\mathbf{S}_{C}$ satisfies ISSC if the sets $\left(S_{i}(X)\right)_{i \in I}, C$ are pairwise disjoint. Clearly, we have that $\{\mathbf{S} \mid \mathbf{S}$ satisfies $\mathrm{SSC}\} \subset\{\mathbf{S}\}$ and $\left\{\mathbf{S}_{C} \mid \mathbf{S}_{C}\right.$ satisfies ISSC $\} \subset\left\{\mathbf{S}_{C}\right\}$.

Note that for $d=1, C^{1}$ conformality means that the maps $S_{i}$ for each $i \in I$ are monotone $C^{1}$ diffeomorphisms. For $d=2, C^{1}$ conformal maps are holomorphic or antiholomorphic. For $d \geq 3$, conformal maps between domains in $\mathbb{R}^{d}$ are of the form $x \mapsto r R(i(x))+a$ where $r>0, a \in \mathbb{R}^{d}, R \in O(d)$ and $i$ is either the identity or an inversion. Here, $O(d)$ denotes the orthogonal group. (A proof of this can be found, for example, in BP92 where it is referred to as Liouville's Theorem (Theorem A.3.7).)

2.3. Infinite IFS and CIFS. So far we have only defined finitely generated IFS and CIFS. We now want to allow the index set $I$ to be a countable infinite subset of $\mathbb{N}$. In order to investigate infinitely generated function systems, we have to introduce the following limit sets. Before stating the next definition, we want to introduce the following notation. Let $I^{n}$ be the family of all finite strings $\mathbf{i}=i_{1}, \ldots, i_{n}$ of length $n$ with entries $i_{j} \in I$ and let $I^{*}:=\bigcup_{n \in \mathbb{N}} I^{n}$ be the family of all finite strings. Also, let $S_{\mathbf{i}}=S_{i_{n}} \circ \ldots \circ S_{i_{1}}$ for $\mathbf{i} \in I^{n}$. We also note that we restrict ourselves to infinitely generated IFS with a bounded attractor. For these, we can always assume that there exists a phase space $X$ (also sometimes called cell) such that $S_{i}(X) \subset X$ for all $i \in I$ and $X$ is the closure of an open set.

Definition 2.2. For an IFS (or CIFS) $\mathbf{S}$ satisfying OSC with phase space $X$ we define

$$
\begin{aligned}
L_{\mathrm{dyn}}(\mathbf{S}) & :=\bigcap_{n \in \mathbb{N}} \bigcup_{\mathbf{i} \in I^{n}} S_{\mathbf{i}}(X) ; \\
L(\mathbf{S}) & :=\overline{L_{\mathrm{dyn}}(\mathbf{S})} ; \\
L_{\mathrm{J}}(\mathbf{S}) & :=\overline{L_{\mathrm{dyn}}(\mathbf{S}) \backslash L_{\mathrm{dyn}}(\mathbf{S}) .}
\end{aligned}
$$

Here, $\overline{L_{\mathrm{dyn}}(\mathbf{S})}$ denotes the closure of $L_{\mathrm{dyn}}(\mathbf{S})$. We call $L(\mathbf{S})$ the limit set, $L_{\mathrm{dyn}}(\mathbf{S})$ the dynamical limit set and $L_{\mathrm{J}}(\mathbf{S})$ the Jørgensen limit set.

Clearly, the set $L_{\mathrm{dyn}}(\mathbf{S})$ can be identified with the set of infinite words $I^{\infty}$ (cf. Hil09 for details). Moreover, note that $L_{\mathrm{dyn}}(\mathbf{S})$ is closed if $\mathbf{S}$ is finitely generated and that $L_{\mathrm{dyn}}(\mathbf{S})$ is in general not closed if $\mathbf{S}$ is infinitely generated. Further note that $L(\mathbf{S})$ is closed by definition. 
Remark 2.3. Note that in [MU96] the set $L_{\mathrm{dyn}}(\mathbf{S})$ is referred to as the "limit set".

Definition 2.4. Let $\mathcal{O}_{\mathbf{S}}(C):=\bigcup_{\mathbf{i} \in I^{*}} S_{\mathbf{i}}(C) \cup C$. For an IIFS (or ICIFS), $\mathbf{S}_{C}$ satisfying IOSC with phase space $X$ and condensation set $C \subset X$, we define

$$
\begin{aligned}
L_{\mathrm{dyn}}\left(\mathbf{S}_{C}\right) & :=\mathcal{O}_{\mathbf{S}}(C) \cup \bigcap_{n \in \mathbb{N}} \bigcup_{\mathbf{i} \in I^{n}} S_{\mathbf{i}}(X)=\mathcal{O}_{\mathbf{S}}(C) \cup L_{\mathrm{dyn}}(\mathbf{S}) ; \\
L\left(\mathbf{S}_{C}\right) & :=\overline{L_{\mathrm{dyn}}\left(\mathbf{S}_{C}\right)}=\overline{\mathcal{O}_{\mathbf{S}}(C) \cup L_{\mathrm{dyn}}(\mathbf{S})}=\overline{\mathcal{O}_{\mathbf{S}}(C)} \cup L(\mathbf{S}) ; \\
L_{\mathrm{J}}\left(\mathbf{S}_{C}\right) & :=\overline{L_{\mathrm{dyn}}\left(\mathbf{S}_{C}\right)} \backslash L_{\mathrm{dyn}}\left(\mathbf{S}_{C}\right) .
\end{aligned}
$$

Lemma 2.5. For an IIFS (or ICIFS), $\mathbf{S}_{C}$ satisfying IOSC with phase space $X$ and condensation set $C \subset X$, we have

$$
L\left(\mathbf{S}_{C}\right)=\mathcal{O}_{\mathbf{S}}(C) \cup L(\mathbf{S}) .
$$

Proof. First note that

$$
\begin{aligned}
& L\left(\mathbf{S}_{C}\right)=\overline{\mathcal{O}_{\mathbf{S}}(C)} \cup L(\mathbf{S}) \\
& =\left(\overline{\mathcal{O}_{\mathbf{S}}(C)} \backslash \mathcal{O}_{\mathbf{S}}(C)\right) \cup \mathcal{O}_{\mathbf{S}}(C) \cup L(\mathbf{S}) \text {. }
\end{aligned}
$$

We will show that $\left(\overline{\mathcal{O}_{\mathbf{S}}(C)} \backslash \mathcal{O}_{\mathbf{S}}(C)\right) \subset L(\mathbf{S})$. Recall that $\mathcal{O}_{\mathbf{S}}(C)=C \cup \bigcup_{\mathbf{i} \in I^{*}} S_{\mathbf{i}}(C)$. Note that for $n \in \mathbb{N} \backslash\{1\}$ we have

$$
\bigcup_{\mathbf{i} \in I^{n}} S_{\mathbf{i}}(C)=\bigcup_{i \in I} \bigcup_{\mathbf{j} \in I^{(n-1)}} S_{i}\left(S_{\mathbf{j}}(C)\right) .
$$

Hence, we have that $\mathcal{O}_{\mathbf{S}}(C)$ satisfies the following inhomogeneous self-similar equation

$$
\begin{aligned}
\mathcal{O}_{\mathbf{S}}(C) & =C \cup \bigcup_{n \in \mathbb{N}} \bigcup_{\mathbf{i} \in I^{n}} S_{\mathbf{i}}(C) \\
& =C \cup \bigcup_{i \in I} S_{i}(C) \cup \bigcup_{\substack{n \in \mathbb{N} \\
n>1}} \bigcup_{i \in I} S_{\mathbf{j} \in I^{(n-1)}}\left(S_{\mathbf{j}}(C)\right) \\
& =C \cup \bigcup_{i \in I} S_{i}(C) \cup \bigcup_{i \in I} \bigcup_{\mathbf{j} \in I^{*}} S_{i}\left(S_{\mathbf{j}}(C)\right) \\
& =C \cup \bigcup_{i \in I} S_{i}\left(C \cup \bigcup_{\mathbf{j} \in I^{*}} S_{\mathbf{j}}(C)\right) \\
& =C \cup \bigcup_{i \in I} S_{i}\left(\mathcal{O}_{\mathbf{S}}(C)\right)
\end{aligned}
$$

Let us now assume that $x \in \overline{\mathcal{O}_{\mathbf{S}}(C)} \backslash \mathcal{O}_{\mathbf{S}}(C)$. We then have

$$
\begin{aligned}
x \in \overline{\mathcal{O}_{\mathbf{S}}(C)} & =\overline{C \cup \bigcup_{i \in I} S_{i}\left(\mathcal{O}_{\mathbf{S}}(C)\right)} \\
& =C \cup \overline{\bigcup_{i \in I} S_{i}\left(\mathcal{O}_{\mathbf{S}}(C)\right)} .
\end{aligned}
$$


Since $x$ is not in $\mathcal{O}_{\mathbf{S}}(C)$ and, in particular, $x$ is not in $C$, we therefore have that

$$
\begin{aligned}
& x \in \overline{\bigcup_{i \in I} S_{i}\left(\mathcal{O}_{\mathbf{S}}(C)\right)}=\overline{\bigcup_{i \in I} S_{i}\left(C \cup \bigcup_{j \in I} S_{j}\left(\mathcal{O}_{\mathbf{S}}(C)\right)\right)} \\
& =\overline{\bigcup_{i \in I} S_{i}(C)} \cup \overline{\bigcup_{i \in I} S_{i}\left(\bigcup_{j \in I} S_{j}\left(\mathcal{O}_{\mathbf{S}}(C)\right)\right)} \\
& =\overline{\bigcup_{i \in I} S_{i}(C)} \cup \overline{\bigcup_{\mathbf{i} \in I^{2}} S_{\mathbf{i}}\left(\mathcal{O}_{\mathbf{S}}(C)\right)} \\
& =\bigcup_{i \in I} S_{i}(C) \cup\left(\overline{\bigcup_{i \in I} S_{i}(C)} \backslash \bigcup_{i \in I} S_{i}(C)\right) \cup \overline{\bigcup_{\mathbf{i} \in I^{2}} S_{\mathbf{i}}\left(\mathcal{O}_{\mathbf{S}}(C)\right)} .
\end{aligned}
$$

Note again that since $x$ is not in $\mathcal{O}_{\mathbf{S}}(C)$ and in particular $x$ is not in $\bigcup_{i \in I} S_{i}(C)$, we therefore have that $x$ is either in $\left(\overline{\bigcup_{i \in I} S_{i}(C)} \backslash \bigcup_{i \in I} S_{i}(C)\right)$ or $\overline{\bigcup_{\mathbf{i} \in I^{2}} S_{\mathbf{i}}\left(\mathcal{O}_{\mathbf{S}}(C)\right)}$. We will first show that

$$
\left(\overline{\bigcup_{i \in I} S_{i}(C)} \backslash \bigcup_{i \in I} S_{i}(C)\right) \subset L(\mathbf{S}) .
$$

Thus assume that $x \in\left(\overline{\bigcup_{i \in I} S_{i}(X)} \backslash \bigcup_{i \in I} S_{i}(C)\right)$. Clearly, there exists a sequence $\left\{x_{i}\right\}_{i \in I}$ which converges to $x$ such that $x_{i} \in S_{i}(C) \subset S_{i}(X)$ for all $i \in I$. If $x \notin \bigcup_{i \in I} S_{i}(X)$, then $x \in\left(\overline{\bigcup_{i \in I} S_{i}(X)} \backslash \bigcup_{i \in I} S_{i}(X)\right) \subset L_{\mathrm{J}}(\mathbf{S})$ and we are finished. Therefore, suppose that $x \in S_{i}(X)$ for some $i \in I$. Then we have that either $x \in L_{\mathrm{dyn}}(\mathbf{S})$ or $x \notin L_{\mathrm{dyn}}(\mathbf{S})$. Hence to complete the proof, assume that $x \notin L_{\mathrm{dyn}}(\mathbf{S})$. Note that for all $\epsilon>0$ there exists $N_{1} \in \mathbb{N}$ such that $\left|x-x_{i}\right|<\epsilon$ for all $i \geq N_{1}$, but since diam $S_{i}(X) \rightarrow 0$ as $i \rightarrow \infty$, there also exists $N_{2} \in \mathbb{N}$ such that $S_{i}(X) \subset B(x, \epsilon)$ for all $i \geq N_{2}$. Hence we can find a sequence $y_{i} \in S_{i}(X) \cap L_{\mathrm{dyn}}(\mathbf{S})$ such that $\left|x-y_{i}\right|<\epsilon$ for all $i \geq \max \left(N_{1}, N_{2}\right)$. This implies that $x \in L_{\mathrm{J}}(\mathbf{S})$ since we have assumed that $x \notin L_{\mathrm{dyn}}(\mathbf{S})$. Thus we have that $x \in L(\mathbf{S})$ or

$$
\begin{aligned}
& x \in \overline{\bigcup_{\mathbf{i} \in I^{2}} S_{\mathbf{i}}\left(\mathcal{O}_{\mathbf{S}}(C)\right)}=\overline{\bigcup_{\mathbf{i} \in I^{2}} S_{\mathbf{i}}\left(C \cup \bigcup_{j \in I} S_{j}\left(\mathcal{O}_{\mathbf{S}}(C)\right)\right)} \\
& =\overline{\bigcup_{\mathbf{i} \in I^{2}} S_{\mathbf{i}}(C)} \cup \overline{\bigcup_{\mathbf{i} \in I^{2}} S_{\mathbf{i}}\left(\bigcup_{j \in I} S_{j}\left(\mathcal{O}_{\mathbf{S}}(C)\right)\right)} \\
& =\overline{\bigcup_{\mathbf{i} \in I^{2}} S_{\mathbf{i}}(C)} \cup \overline{\bigcup_{\mathbf{i} \in I^{3}} S_{\mathbf{i}}\left(\mathcal{O}_{\mathbf{S}}(C)\right)} \\
& =\bigcup_{\mathbf{i} \in I^{2}} S_{\mathbf{i}}(C) \cup\left(\overline{\bigcup_{\mathbf{i} \in I^{2}} S_{\mathbf{i}}(C)} \backslash \bigcup_{\mathbf{i} \in I^{2}} S_{\mathbf{i}}(C)\right) \cup \overline{\bigcup_{\mathbf{i} \in I^{3}} S_{\mathbf{i}}\left(\mathcal{O}_{\mathbf{S}}(C)\right)} \\
& \subset \bigcup_{\mathbf{i} \in I^{2}} S_{\mathbf{i}}(C) \cup L(\mathbf{S}) \cup \overline{\bigcup_{\mathbf{i} \in I^{3}} S_{\mathbf{i}}\left(\mathcal{O}_{\mathbf{S}}(C)\right)} \text {. }
\end{aligned}
$$


Here, in the final equation we have used an argument as before to conclude that $\left(\overline{\bigcup_{\mathbf{i} \in I^{2}} S_{\mathbf{i}}(C)} \backslash \bigcup_{\mathbf{i} \in I^{2}} S_{\mathbf{i}}(C)\right) \subset L(\mathbf{S})$. Therefore, since $x$ is not in $\mathcal{O}_{\mathbf{S}}(C)$ we have that $x \in L(\mathbf{S})$ or $x \in \overline{\bigcup_{\mathbf{i} \in I^{3}} S_{\mathbf{i}}\left(\mathcal{O}_{\mathbf{S}}(C)\right)}$. Hence, by iteration we have that

$$
x \in L(\mathbf{S}) \text { or } x \in \overline{\bigcup_{\mathbf{i} \in I^{n}} S_{\mathbf{i}}\left(\mathcal{O}_{\mathbf{S}}(C)\right)} \text { for all } n \in \mathbb{N} .
$$

Finally, note that

$$
\overline{\bigcup_{\mathbf{i} \in I^{n}} S_{\mathbf{i}}\left(\mathcal{O}_{\mathbf{S}}(C)\right)} \subset \overline{\bigcup_{\mathbf{i} \in I^{n}} S_{\mathbf{i}}(X)} \text { for all } n \in \mathbb{N} .
$$

Therefore

$$
x \in L(\mathbf{S}) \text { or } x \in \overline{\bigcup_{\mathbf{i} \in I^{n}} S_{\mathbf{i}}(X)} \text { for all } n \in \mathbb{N} \text {. }
$$

This implies that

$$
x \in L(\mathbf{S}) \text { or } x \in \overline{\bigcap_{n \in \mathbb{N}} \bigcup_{\mathbf{i} \in I^{n}} S_{\mathbf{i}}(X)} \subset L(\mathbf{S}) .
$$

Hence we conclude that

$$
\left(\overline{\mathcal{O}_{\mathbf{S}}(C)} \backslash \mathcal{O}_{\mathbf{S}}(C)\right) \subset L(\mathbf{S})
$$

\section{Teichmüller SPACE FOR (FInite AND infinite) ITERATED FUNCTION SYSTEMS}

In this section we will introduce the notion of Teichmüller space for IFS. For any fixed (finite or infinite) alphabet $I$, let $\operatorname{IFS}(I)$ denote the space of all iterated function systems with alphabet $I$ and let $\operatorname{IIFS}(I)$ denote the space of all inhomogeneous iterated function systems with alphabet $I$. Since contracting similarities on $\mathbb{R}^{d}$ are of the form $S_{i} x=r_{i} R_{i} x+a_{i}$, with $r_{i} \in(0,1), R_{i}$ is an orthogonal matrix and $a_{i} \in \mathbb{R}^{d}$ for $i \in I$, we can represent each contracting similarity $S_{i}$ by the point $\left(a_{i}, r_{i}, R_{i}\right)$ in the space $\mathbb{R}^{d} \times(0,1) \times O(d)$, where $O(d)$ denotes the orthogonal group of degree $d$ over $\mathbb{R}^{d}$. A natural way to change the properties of a given IFS is to change the contraction ratios $r_{i}$ or additive constants $a_{i}$ or orthogonal matrices $R_{i}$ of all or some of the contracting similarities. For example, if an IFS satisfies SSC, changing contraction ratios in a particular way will obviously lead to an IFS which does not satisfy SSC any longer. Motivated by this, we define the deformation space or Teichmüller space for IFS $T(\operatorname{IFS}(I))$ by

$$
T(\operatorname{IFS}(I)):=\{\mathbf{S} \in \operatorname{IFS}(I) \mid \mathbf{S} \text { satisfies } \operatorname{SSC}\} .
$$

It is natural to study the boundary $\partial T(\operatorname{IFS}(I))$ of $T(\operatorname{IFS}(I))$.

Definition 3.1. We say that a sequence $\left\{\mathbf{S}_{k}\right\}_{k \in \mathbb{N}}=\left\{\left\{S_{i, k}\right\}_{i \in I}\right\}_{k \in \mathbb{N}}$ in $T(\operatorname{IFS}(I))$ converges pointwise to $\mathbf{S}=\left\{S_{i}\right\}_{i \in I} \in T(\operatorname{IFS}(I)) \cup \partial T(\operatorname{IFS}(I))$ if and only if $\left(a_{i, k}\right.$, $\left.r_{i, k}, R_{i, k}\right) \rightarrow\left(a_{i}, r_{i}, R_{i}\right)$ as $k \rightarrow \infty$ for all $i \in I$.

Let $\overline{\mathbb{R}}$ denote the closure of $\mathbb{R}$, i.e. $\overline{\mathbb{R}}=\mathbb{R} \cup\{ \pm \infty\}$. Clearly, we can have in Definition 3.1 that $a_{i, k} \rightarrow a_{i}$ with $a_{i} \in \overline{\mathbb{R}}^{d} \backslash \mathbb{R}^{d}$ as $k \rightarrow \infty$. This is obviously an undesirable situation and we will not consider such cases for the rest of the paper. However, before stating our main theorem, we want to discuss two particular cases 
of interest. Therefore, define an extended condensation transformation $\Xi: \overline{\mathbb{R}}^{d} \rightarrow \overline{\mathbb{R}}^{d}$ by $\Xi x=a$ with $a \in \overline{\mathbb{R}}^{d}$ and $\|a\|=\infty$. Let $\boldsymbol{\Xi}=\left\{\Xi_{i}\right\}_{i \in I}$ be a set of extended condensation transformations on $\overline{\mathbb{R}}^{d}$. Let $\boldsymbol{\Psi}=\left\{\psi_{i}\right\}_{i \in I}$ be a set of isometries on $\mathbb{R}^{d}$. Let $\operatorname{ExIFS}_{\overline{\mathbb{R}}^{d}}(I):=\left\{\{\psi\} \cup\left\{\Xi_{j}\right\}_{j \in J}|\psi \in \Psi, J \subsetneq I| J,|=| I \mid-1\right\}$, where $|\cdot|$ denotes the cardinality of a set. Now assume without loss of generality that $1 \in I$. Let $\mathbf{S}$ be in $T(\operatorname{IFS}(I))$ and rewrite $\mathbf{S}$ as $\mathbf{S}=\left\{S_{i}\right\}_{i \in I \backslash\{1\}} \cup\left\{S_{1}\right\}$ with $S_{i} x=r_{i} R_{i} x+a_{i}$, $r_{i} \in(0,1), R_{i} \in O(d), a_{i} \in \mathbb{R}^{d}$ for $i \in I$. By letting $r_{1}$ tend to 1 we change $\mathbf{S}$ so that $\mathbf{S}$ converges pointwise to a system in $\operatorname{ExIFS}_{\overline{\mathbb{R}}^{d}}(I)$. Since $S_{1}$ tends to the isometry transformation $\hat{S}_{1} x=R_{1} x+a_{1}$ when $r_{1}$ tends to 1 and since the sets $\left\{S_{i}(L(\mathbf{S}))\right\}_{i \in I \backslash\{1\}}, S_{1}(L(\mathbf{S}))$ have to stay pairwise disjoint while we increase $r_{1}$ to 1 we need to let $r_{i} \rightarrow 0$ and $\left\|a_{i}\right\| \rightarrow \infty$ for all $i \in I \backslash\{1\}$ as $r_{1} \rightarrow 1$. Hence $\mathbf{S}$ converges pointwise to $\left\{\tilde{S}_{i}\right\}_{i \in I \backslash\{1\}} \cup\left\{\hat{S}_{1}\right\}$ such that $\tilde{S}_{i}: \overline{\mathbb{R}}^{d} \rightarrow \overline{\mathbb{R}}^{d}$ is defined by $\tilde{S}_{i} x=a_{i}$ with $\left\|a_{i}\right\|=\infty$ for all $i \in I \backslash\{1\}$ and $\hat{S}_{1} x=R_{1} x+a_{1}$. Note that when $|I|=1$ the system consists of one isometry and there are no extended condensation transformations. However, such systems are trivial and therefore for the rest of the paper we assume that $|I|>1$.

Recall that for each IFS or IIFS on $\mathbb{R}^{d}$ the limit set is bounded. However, a sequence $\left\{\mathbf{S}_{k}\right\}_{k \in \mathbb{N}} \in T(\operatorname{IFS}(I))$ may converge to a system $\mathbf{S} \in \partial T(\operatorname{IFS}(I))$ such that $L(\mathbf{S})$ is unbounded, namely if $\operatorname{diam} L\left(\mathbf{S}_{k}\right) \rightarrow \infty$ as $k \rightarrow \infty$. Therefore, there are IFS and IIFS on $\overline{\mathbb{R}}^{d}$ with unbounded limit set.

Definition 3.2. Define the essential boundary of $T(\operatorname{IFS}(I))$ by

$$
\partial_{\text {ess }} T(\operatorname{IFS}(I)):=\partial T(\operatorname{IFS}(I)) \cap\left\{\mathbf{S} \in \partial T(\operatorname{IFS}(I)) \mid a_{i} \notin \overline{\mathbb{R}}^{d} \backslash \mathbb{R}^{d}\right\} .
$$

Let $\operatorname{IFS}_{p \in \mathbb{R}^{d}}(I):=\left\{\mathbf{S} \in \operatorname{IFS}(I) \mid L(\mathbf{S})=\{p\}, p \in \mathbb{R}^{d}\right\}$ and $\operatorname{IIFS}_{p \in \mathbb{R}^{d}}(I):=\left\{\mathbf{S}_{C} \in\right.$ $\left.\operatorname{IIFS}(I) \mid L\left(\mathbf{S}_{C}\right)=\{p\}, p \in \mathbb{R}^{d}\right\}$.

Definition 3.3. A set $X$ is star-shaped with center $c \in X$, if for every $x \in X$ the line segment $\{c+t(x-c), t \in[0,1]\} \subset X$.

In order to prove our main results in this section we need to consider IFS satisfying OSC with an open set $U$ being star-shaped. The next theorem gives a description of $\partial_{\text {ess }} T(\operatorname{IFS}(I))$.

Theorem 3.4. For $|I|>1$ we have,

(1) $\{\mathbf{S} \in I F S(I) \mid \mathbf{S}$ satisfies OSC but not SSC with a star-shaped open set $U\}$ $\subset \partial_{\text {ess }} T(\operatorname{IFS}(I))$,

(2) $\partial_{\text {ess }} T(\operatorname{IFS}(I)) \backslash\{\mathbf{S} \in I F S(I) \mid \mathbf{S}$ satisfies OSC but not $S S C\} \subset\left\{\mathbf{S}_{C} \in\right.$ $I I F S(J) \mid \mathbf{S}_{C}$ satisfies IOSC and $\left.J \subsetneq I\right\} \cup I F S_{p \in \mathbb{R}^{d}}(I) \cup \bigcup_{J \subsetneq I} \operatorname{IIFS}_{p \in \mathbb{R}^{d}}(J)$.

Remark 3.5. From now on, we identify a limiting system $\mathbf{S} \in \partial_{\text {ess }} T(\operatorname{IFS}(I))$ of the form $\mathbf{S}=\left\{S_{i}\right\}_{i \in I_{1}} \cup\left\{S_{i}\right\}_{i \in I_{2}}$, where $I_{1} \cup I_{2}=I$ and $r_{i}=0$ if and only if $i \in I_{2}$ with the system $\left(\left\{S_{i}\right\}_{i \in I_{1}}, \bigcup_{i \in I_{2}}\left\{a_{i}\right\}\right) \in \operatorname{IIFS}\left(I_{1}\right)$.

Proof. (1) We believe that this fact is folklore. It can be seen as follows. To prove that $\{\mathbf{S} \in \operatorname{IFS}(I) \mid \mathbf{S}$ satisfies OSC but not SSC with a star-shaped open set $U\} \subset$ $\partial_{\text {ess }} T(\operatorname{IFS}(I))$ it suffices to show that for every $\mathbf{S} \in\{\mathbf{S} \in \operatorname{IFS}(I) \mid \mathbf{S}$ satisfies OSC but not SSC with a star-shaped open set $U\}$ there exists a sequence $\left\{\mathbf{S}_{k}\right\}_{k \in \mathbb{N}}$ in $T(\operatorname{IFS}(I))$ which converges pointwise to $\mathbf{S}$. Fix an IFS $\mathbf{S}=\left\{S_{i}\right\}_{i \in I}$ satisfying the OSC but not SSC and let $U$ be such an open star-shaped set. For $k \in \mathbb{N}$ 
sufficiently large and each $i \in I$ there exists a contracting similarity $S_{i, k}: \mathbb{R}^{d} \rightarrow \mathbb{R}^{d}$ such that $r_{i, k}=r_{i}-\frac{1}{k}, R_{i, k}=R_{i}$ and $a_{i, k}$ is chosen such that $S_{i, k}(\bar{U}) \subset S_{i}(U)$. Note that given $r_{i, k}=r_{i}-\frac{1}{k}$ we can always find a corresponding $a_{i, k}$ such that $S_{i, k}(\bar{U}) \subset S_{i}(U)$, since $U$ is star-shaped and therefore $\operatorname{diam} S_{i, k}(\bar{U})<\operatorname{diam} S_{i}(U)$. Clearly, we also have that $\left\|a_{i}-a_{i, k}\right\| \rightarrow 0$ as $k \rightarrow \infty$, if $S_{i, k}(\bar{U}) \subset S_{i}(U)$ holds for all sufficiently large $k$. Since $L\left(\mathbf{S}_{k}\right) \subseteq \bar{U}$ we then have for all sufficiently large $k$ that $S_{i, k}\left(L\left(\mathbf{S}_{k}\right)\right) \cap S_{j, k}\left(L\left(\mathbf{S}_{k}\right)\right) \subseteq S_{i, k}(\bar{U}) \cap S_{j, k}(\bar{U}) \subset S_{i}(U) \cap S_{j}(U)=\emptyset$ if $i \neq j$ and therefore each $\mathbf{S}_{k}$ satisfies SSC. Hence, $\left\{\mathbf{S}_{k}\right\}_{k \in \mathbb{N}} \in T(\operatorname{IFS}(I))$, and letting $k \rightarrow \infty$ gives the desired result.

(2) Without loss of generality assume that $1 \in I$. Let $\mathbf{S}$ be in $T(\operatorname{IFS}(I))$ and rewrite $\mathbf{S}$ as $\mathbf{S}=\left\{S_{i}\right\}_{i \in I \backslash\{1\}} \cup\left\{S_{1}\right\}$ with $S_{i} x=r_{i} R_{i} x+a_{i}, r_{i} \in(0,1), R_{i} \in O(d)$, $a_{i} \in \mathbb{R}^{d}$ for $i \in I$. By letting $r_{1}$ tend to 0 we change $\mathbf{S}$ so that $\mathbf{S}$ converges pointwise to a system in $\partial_{\text {ess }} T(\operatorname{IFS}(I)) \backslash\{\mathbf{S} \in \operatorname{IFS}(I) \mid \mathbf{S}$ satisfies OSC but not SSC $\} \subset\left\{\mathbf{S}_{C} \in\right.$ $\operatorname{IIFS}(J) \mid \mathbf{S}_{C}$ satisfies IOSC and $\left.J \subsetneq I\right\}$ in the following way. Since $S_{1}$ tends to the condensation transformation $\tilde{S}_{1} x=a_{1}$ when $r_{1}$ tends to 0 and since clearly the sets $\left\{S_{i}\left(L\left(\mathbf{S}_{\left\{a_{1}\right\}}\right)\right)\right\}_{i \in I \backslash\{1\}},\left\{a_{1}\right\}$ are pairwise disjoint, we therefore have that $\mathbf{S}$ converges pointwise to $\mathbf{S}_{\left\{a_{1}\right\}} \in\left\{\mathbf{S}_{C} \in \operatorname{IIFS}(I \backslash\{1\}) \mid \mathbf{S}_{C}\right.$ satisfies ISSC $\} \subset\left\{\mathbf{S}_{C} \in\right.$ $\operatorname{IIFS}(J) \mid \mathbf{S}_{C}$ satisfies IOSC and $\left.J \subsetneq I\right\}$ as $r_{1}$ tends to 0 . However we can also have that $a_{1} \rightarrow a_{i}$ for some $i \in I \backslash\{1\}$ as $r_{1} \rightarrow 0$. In this case we would have that $\mathbf{S}$ converges pointwise to $\mathbf{S}_{\left\{a_{i}\right\}} \in\left\{\mathbf{S}_{C} \in \operatorname{IIFS}(I \backslash\{1\}) \mid \mathbf{S}_{C}\right.$ satisfies IOSC $\} \subset\left\{\mathbf{S}_{C} \in\right.$ $\operatorname{IIFS}(J) \mid \mathbf{S}_{C}$ satisfies IOSC and $\left.J \subsetneq I\right\}$ as $r_{1}$ tends to 0 .

Let $\left\{\mathbf{S}_{k}\right\}_{k \in \mathbb{N}}=\left\{\left\{S_{i, k}\right\}_{i \in I}\right\}_{k \in \mathbb{N}}$ be a sequence in $T(\operatorname{IFS}(I))$ which converges pointwise to $\mathbf{S}=\left\{S_{i}\right\}_{i \in I}$ with $S_{i}$ being contracting similarities sharing the same fixed point $p \in \mathbb{R}^{d}$ for all $i \in I$. Then clearly, diam $L\left(\mathbf{S}_{k}\right) \rightarrow 0$. Note that in this case $\mathbf{S}$ does not satisfy OSC and $L(\mathbf{S})=\{p\}$.

Without loss of generality assume that $1 \in I$. Let $\left\{\mathbf{S}_{k}\right\}_{k \in \mathbb{N}}=\left\{\left\{S_{i, k}\right\}_{i \in I}\right\}_{k \in \mathbb{N}}$ be a sequence in $T(\operatorname{IFS}(I))$ which converges pointwise to $\left\{S_{1}\right\} \cup\left\{S_{i}\right\}_{i \in I \backslash\{1\}}$ with $S_{1} x=p$ and $S_{i}$ being contracting similarities sharing the same fixed point $p$ for all $i \in I \backslash\{1\}$ and $p \in \mathbb{R}^{d}$. Then clearly, diam $L\left(\mathbf{S}_{k}\right) \rightarrow 0$ and $\mathbf{S}_{k}$ converges pointwise to IIFS $\mathbf{S}_{\{p\}}$ as $k \rightarrow \infty$. Note that in this case $\mathbf{S}_{\{p\}}$ does not satisfy IOSC and $L\left(\mathbf{S}_{\{p\}}\right)=\{p\}$.

Remark 3.6. Note that in KS08 the boundary of moduli space consists only of IFS satisfying OSC and IIFS satisfying IOSC. In particular, there are no systems from the set $\operatorname{IFS}_{p \in \mathbb{R}^{d}}(I) \cup \bigcup_{J \subsetneq I} \operatorname{IIFS}_{p \in \mathbb{R}^{d}}(J)$. This discrepancy is due to the fact that in [KS08] the moduli space for IFS is seen from the point of view of CIFS. Namely, in [KS08, IFS are acting on a phase space $X$ which is kept fixed and therefore this phase space $X$ cannot collapse to a point. This is exactly what happens if the sequence of IFS converges to a system in $\operatorname{IFS}_{p \in \mathbb{R}^{d}}(I) \cup \bigcup_{J \subsetneq I} \operatorname{IIFS}_{p \in \mathbb{R}^{d}}(J)$ in Theorem 3.4

If we keep the phase space $X$ fixed, then we can restate Theorem 3.4 as follows.

Theorem 3.7. Assume that the phase space $X$ is star-shaped. Then for $|I|>1$ we have,

(1) $\{\mathbf{S} \in \operatorname{IFS}(X, I) \mid \mathbf{S}$ satisfies $O S C$ but not $S S C\} \subset \partial_{\text {ess }} T(\operatorname{IFS}(X, I))$,

(2) $\partial_{\text {ess }} T(\operatorname{IFS}(X, I)) \backslash\{\mathbf{S} \in \operatorname{IFS}(X, I) \mid \mathbf{S}$ satisfies OSC but not $S S C\} \subset\left\{\mathbf{S}_{C} \in\right.$ $\operatorname{IIFS}(X, J) \mid \mathbf{S}_{C}$ satisfies IOSC and $\left.J \subsetneq I\right\}$. 
Example 3.8. Let us consider $T(\operatorname{IFS}(I))$ for $I=\{1,2,3\}$. In this case we can represent each contracting similarity by a point in the space $\mathbb{R} \times(0,1) \times\{1,-1\}$ and therefore the parameter space for all three contracting similarities will have a dimension which is too big to visualize. Hence we fix $S_{1}$ and $S_{3}$ and vary only $S_{2}$ so that we can draw a two dimensional picture of the parameter space. Let $\mathbf{S}=$ $\left\{S_{1}, S_{2}, S_{3}: \mathbb{R} \rightarrow \mathbb{R}\right\}$ with $S_{1}(x)=1 / 3 x, S_{3}(x)=1 / 3 x+2 / 3$ and $S_{2}(x)=r x+a$, $r \in(0,1), a \in \mathbb{R}$. Under these assumptions, one can easily check which pairs of $(r, a)$ give rise to an element in Teichmüller space. More precisely, there are three different situations to check.

Firstly, for $a \in(0,1)$ it is easily seen that $\mathbf{S}$ satisfies OSC if and only if $r \in$ $(0,1 / 3], a \in[1 / 3,2 / 3], a+r \leq 2 / 3$.

Secondly, for $a \geq 1$ one easily calculates that $\mathbf{S}$ satisfies OSC if and only if $r \in(0,1 / 3], a \geq 1$, with $r \leq 1-\frac{a}{3 a-2}$ and $r \leq 1-\frac{a}{2}$.

Finally, for $a \leq 0$ one easily calculates that $\mathbf{S}$ satisfies OSC if and only if $r \in$ $(0,1 / 3], a \leq 0$, with $r \leq 1+a$ and $r+a \leq \frac{a}{3(1-r)}$.

Therefore the interior of the middle triangle in Figure 3.0.1 represents the part of Teichmüller space for $a \in(0,1)$, the interior of the region on the right-hand side represents the part of Teichmüller space for $a \geq 1$ and the interior of the region on the left-hand side represents the part of Teichmüller space for $a \leq 0$. In particular, the part of the boundary which consists of IIFS is exactly on the real line, i.e. $r=0$, and the part of the boundary which satisfies OSC is the remainder of the boundary of these three regions. Note, since we keep $S_{1}$ and $S_{3}$ fixed, we do not have any pathological cases on the boundary of this part of Teichmüller space.

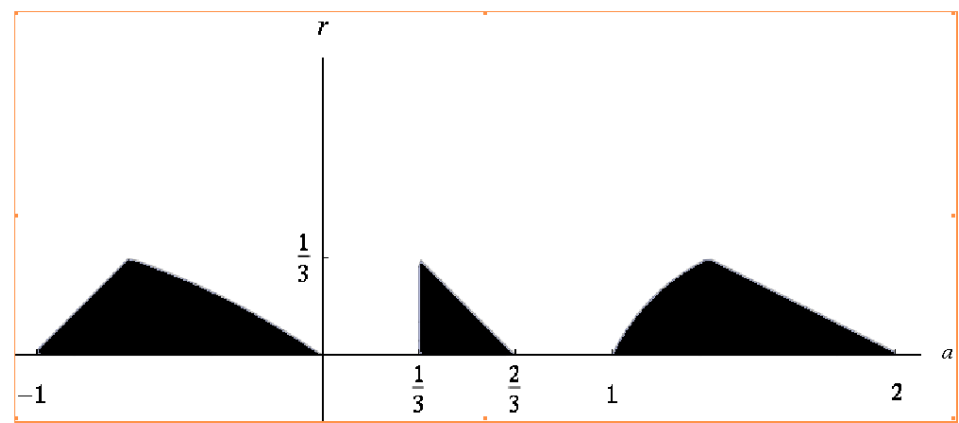

FiguRE 3.0.1. Teichmüller space for $S_{1}(x)=1 / 3 x, S_{3}(x)=1 / 3 x+2 / 3$ and $S_{2}(x)=r x+a$.

Example 3.9. Let us give another example. This time, let $I=\{1,2\}$ and again fix $S_{1}(x)=1 / 3 x$ and vary $S_{2}(x)=r x+a, r \in(0,1), a \in \mathbb{R}$. One easily verifies that $\mathbf{S}$ satisfies SSC for all $r \in(0,2 / 3)$ and all $a \in \mathbb{R} \backslash\{0\}$. The corresponding part of Teichmüller space is represented in Figure 3.0.2. The part of the boundary consisting of IFS satisfying OSC (but not SSC) is on the line $r=\frac{2}{3}$ and the part of the boundary consisting of IIFS is exactly on the real line, i.e. $r=0$. However, unlike in Example [3.8, there is a part of the boundary consisting of IFS with $S_{2}(x)=r x$, i.e. $a=0$. Note that these systems are actually in $\operatorname{IFS}_{p \in \mathbb{R}^{d}}(I)$ and when additionally $r=0$ in $\bigcup_{J \subsetneq I} \operatorname{IIFS}_{p \in \mathbb{R}^{d}}(J)$. Furthermore, in this example one 
can see how systems with unbounded limit set occur. These are represented by the boundary "at infinity", i.e. for $a= \pm \infty$.

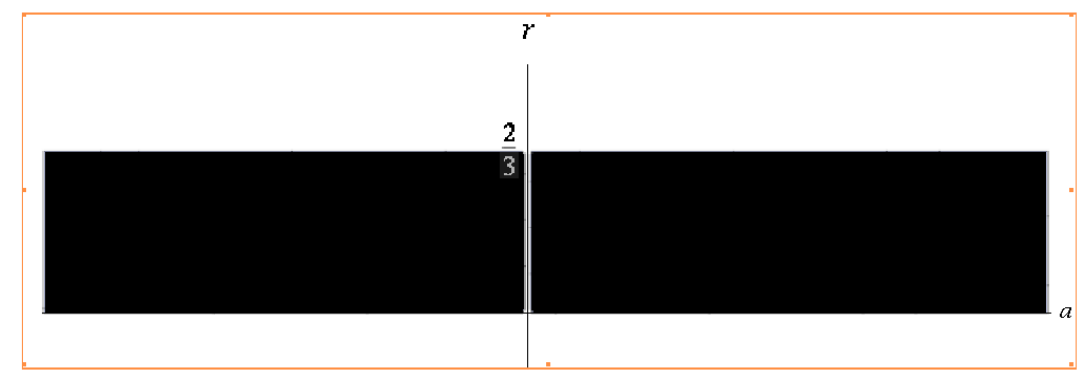

FiguRE 3.0.2. Teichmüller space for $S_{1}(x)=1 / 3 x$ and $S_{2}(x)=r x+a$.

Example 3.10. Note that in Theorem 3.4 the part of the boundary $\partial_{\mathrm{ess}}^{\text {iosc }} T(\operatorname{IFS}(I))$ consists of IIFS with condensation set $C$ being a countable set. We now construct an example where we deform an affine IFS and obtain in the limit an IIFS with a nontrivial condensation set $C$. This indicates that the boundary of Teichmüller space for affine IFS includes IIFS with more interesting condensation sets and we study this in $\mathrm{HSa}$. Let $\left\{\mathbf{S}_{k}\right\}_{k \in \mathbb{N}}=\left\{S_{1}, S_{2}, S_{3}, S_{4, k}\right\}_{k \in \mathbb{N}}$ with $S_{1}(x, y)=\left(\frac{x}{3}+2, \frac{y}{3}+2\right)$, $S_{2}(x, y)=\left(\frac{x}{3}, \frac{y}{3}+2\right), S_{3}(x, y)=\left(\frac{x}{3}+2, \frac{y}{3}\right)$ and $S_{4, k}(x, y)=\left(\frac{x}{3}, \frac{y}{3 k}\right)$. We then have that $\left\{\mathbf{S}_{k}\right\}_{k \in \mathbb{N}}$ converges pointwise to $\mathbf{S}=\left(\left\{S_{1}, S_{2}, S_{3}\right\}, C\right)$ as $k \rightarrow \infty$ where $C$ is the middle third Cantor set.

3.1. Metrics on $T(\operatorname{IFS}(I))$. For ease of exposition we write

$$
\begin{aligned}
\partial_{\text {ess }} T(\operatorname{IFS}(I))=\partial_{\mathrm{ess}}^{\text {osc }} T(\operatorname{IFS}(I)) \cup \partial_{\mathrm{ess}}^{\text {iosc }} T(\operatorname{IFS}(I)) \\
\cup \partial_{\mathrm{ess}}^{\text {nosc }} T(\operatorname{IFS}(I)) \cup \partial_{\mathrm{ess}}^{\text {niosc }} T(\operatorname{IFS}(I)),
\end{aligned}
$$

where

$$
\begin{aligned}
\partial_{\mathrm{ess}}^{\mathrm{osc}} T(\operatorname{IFS}(I)): & =\{\mathbf{S} \in \operatorname{IFS}(I) \mid \mathbf{S} \text { satisfies OSC but not } \operatorname{SSC}\} \cap \partial_{\mathrm{ess}} T(\operatorname{IFS}(I)), \\
\partial_{\mathrm{ess}}^{\mathrm{iosc}} T(\operatorname{IFS}(I)):= & \left\{\mathbf{S}_{C} \in \operatorname{IIFS}(J) \mid \mathbf{S}_{C} \text { satisfies IOSC and } J \subsetneq I\right\} \cap \partial_{\mathrm{ess}} T(\operatorname{IFS}(I)), \\
& \partial_{\mathrm{ess}}^{\text {nosc }} T(\operatorname{IFS}(I)):=\operatorname{IFS}_{p \in \mathbb{R}^{d}}(I) \cap \partial_{\mathrm{ess}} T(\operatorname{IFS}(I))
\end{aligned}
$$

and

$$
\partial_{\mathrm{ess}}^{\mathrm{niosc}} T(\operatorname{IFS}(I)):=\bigcup_{J \subsetneq I} \operatorname{IIFS}_{p \in \mathbb{R}^{d}}(J) \cap \partial_{\mathrm{ess}} T(\operatorname{IFS}(I)) .
$$

For the moment we assume that the alphabet $I$ is finite. We will introduce two different metrics on $T(\operatorname{IFS}(I))$. We will use these metrics to analyze how far different parts of the essential boundary $\partial_{\text {ess }} T(\operatorname{IFS}(I))$ are from any given IFS $\tilde{\mathbf{S}} \in T(\operatorname{IFS}(I))$. More precisely, we are interested in the following distance. Let $\tilde{\mathbf{S}}=\left\{\tilde{S}_{i}\right\}_{i \in I}$ be any IFS in $T(\operatorname{IFS}(I))$ and let $\left\{\mathbf{S}_{k}\right\}_{k \in \mathbb{N}}=\left\{\left\{S_{i, k}\right\}_{i \in I}\right\}_{k \in \mathbb{N}}$ be a sequence of IFS in $T(\operatorname{IFS}(I))$ which converges pointwise to $\mathbf{S}$ in $\partial_{\text {ess }} T(\operatorname{IFS}(I))$. Then we define the distance between $\tilde{\mathbf{S}}$ and $\mathbf{S}$ by

$$
\operatorname{dist}(\tilde{\mathbf{S}}, \mathbf{S}):=\lim _{k \rightarrow \infty} d_{T(\operatorname{IfS}(I))}\left(\tilde{\mathbf{S}}, \mathbf{S}_{k}\right),
$$


where for all $k \in \mathbb{N}$ we define the distance $d_{T(\operatorname{IFS}(I))}$ between $\tilde{\mathbf{S}}$ and $\mathbf{S}_{k}$ by

$$
d_{T(\operatorname{IFS}(I))}\left(\tilde{\mathbf{S}}, \mathbf{S}_{k}\right):=\sum_{i \in I}\left(d\left(\tilde{S}_{i}, S_{i, k}\right)\right) .
$$

It will become clear that $d_{T(\operatorname{IFS}(I))}\left(\tilde{\mathbf{S}}, \mathbf{S}_{k}\right)$ does not depend on the choice of $\left\{\mathbf{S}_{k}\right\}_{k \in \mathbb{N}}$. Therefore it suffices to define the distance between any two contracting similarities $S_{1}$ and $S_{2}$. As above, let $S_{i} x=r_{i} R_{i} x+a_{i}, r_{i} \in(0,1), R_{i} \in O(d)$ and $a_{i} \in \mathbb{R}^{d}$ for $i \in I$ and recall that we can represent each contracting similarity $S_{i}$ by the point $\left(a_{i}, r_{i}, R_{i}\right)$ in the space $\mathbb{R}^{d} \times(0,1) \times O(d)$. Note that $\mathbb{R}^{d} \times(0,1) \times O(d) \subset$ $\mathbb{R}^{d} \times(0, \infty) \times O(d)$ and hence we can interpret $\mathbb{R}^{d} \times(0,1)$ either as a subspace of $d+1$ dimensional hyperbolic space $\mathbb{H}^{d+1}$ and equip it with the usual hyperbolic metric $d_{\text {hyp }}$ or as a subspace of $\mathbb{R}^{d} \times \mathbb{R}^{+}$and equip it with the Manhattan metric $d_{\text {man }}$. We will show that the choice of the metric for $\mathbb{R}^{d} \times(0,1)$ is crucial in determining how far the part of the essential boundary $\partial_{\mathrm{ess}}^{\text {iosc }} T(\operatorname{IFS}(I)) \cup \partial_{\mathrm{ess}}^{\text {niosc }} T(\operatorname{IFS}(I))$ is from the interior of $T(\operatorname{IFS}(I))$. It is well known that $O(d)$ is a real compact Lie group. Hence it is clearly possible to equip $O(d)$ with a metric $d_{O(d)}$ such that $\operatorname{diam}(O(d)) \leq 1$, i.e. $d_{O(d)}(A, B) \leq 1$ for all $A, B \in O(d)$. We now define the distance between two contracting similarities $S_{1}$ and $S_{2}$ in the following two ways. Firstly, we consider

$$
d_{h}\left(S_{1}, S_{2}\right):=d_{\text {hyp }}\left(\left(a_{1}, r_{1}\right),\left(a_{2}, r_{2}\right)\right)+d_{O(d)}\left(R_{1}, R_{2}\right) .
$$

Clearly (3.3) defines a metric on the space $\mathbb{R}^{d} \times(0,1) \times O(d)$ and hence (3.2) with $d:=d_{h}$ defines a metric on the space $T(\operatorname{IFS}(I))$ which we call the hyperbolic metric $d_{T(\operatorname{IFS}(I))}$. From Theorem 3.4 we have that if $\mathbf{S} \in \partial_{\text {ess }} T(\operatorname{IFS}(I))$, then $\mathbf{S}$ is either in $\partial_{\text {ess }}^{\text {osc }} T(\operatorname{IFS}(I)) \cup \partial_{\text {ess }}^{\text {nosc }} T(\operatorname{IFS}(I))$ or in $\partial_{\text {ess }}^{\text {iosc }} T(\operatorname{IFS}(I)) \cup \partial_{\text {ess }}^{\text {niosc }} T(\operatorname{IFS}(I))$. In the first case we clearly have that $\operatorname{dist}(\tilde{\mathbf{S}}, \mathbf{S})<\infty$ independent of the choice of $\left\{\mathbf{S}_{k}\right\}_{k \in \mathbb{N}}$. In the second case we have that at least one of the contracting similarities $S_{i, k}$ in the sequence of IFS $\left\{\mathbf{S}_{k}\right\}_{k \in \mathbb{N}}$ in $T(\operatorname{IFS}(I))$ converges pointwise to a condensation transformation. In particular we then have that $r_{i, k} \rightarrow 0$ for some $i \in I$ as $k \rightarrow \infty$ and therefore $\operatorname{dist}(\tilde{\mathbf{S}}, \mathbf{S})$ diverges since we compute:

$$
\begin{aligned}
\operatorname{dist}(\tilde{\mathbf{S}}, \mathbf{S}) & =\lim _{k \rightarrow \infty} d_{T(\operatorname{IFS}(I))}\left(\tilde{\mathbf{S}}, \mathbf{S}_{k}\right) \\
& =\lim _{k \rightarrow \infty} \sum_{i \in I}\left(d_{h}\left(\tilde{S}_{i}, S_{i, k}\right)\right) \\
& =\sum_{i \in I} \lim _{k \rightarrow \infty}\left(d_{h}\left(\tilde{S}_{i}, S_{i, k}\right)\right) \\
& \geq \lim _{k \rightarrow \infty}\left(d_{h}\left(\tilde{S}_{i}, S_{i, k}\right)\right), \quad \text { where } i \in I \text { is such that } r_{i, k} \rightarrow 0 \text { as } k \rightarrow \infty \\
& =\lim _{k \rightarrow \infty}\left(d_{\text {hyp }}\left(\left(\tilde{a}_{i}, \tilde{r}_{i}\right),\left(a_{i, k}, r_{i, k}\right)\right)+d_{O(d)}\left(\tilde{R}_{i}, R_{i, k}\right)\right) \\
& \geq \lim _{k \rightarrow \infty}\left(d_{\text {hyp }}\left(\tilde{a}_{i}, \tilde{r}_{i}\right),\left(a_{i, k}, r_{i, k}\right)\right) \\
& \geq \lim _{k \rightarrow \infty}\left(d_{\text {hyp }}\left(0, \tilde{r}_{i}\right),\left(0, r_{i, k}\right)\right) \\
& =\lim _{k \rightarrow \infty}\left|\log \tilde{r}_{i}-\log r_{i, k}\right| \\
& =\infty .
\end{aligned}
$$

Note that if $r_{i, k} \rightarrow 0$ for some $i \in I$ as $k \rightarrow \infty$, then the corresponding $R_{i, k}$ do not need to converge as $k \rightarrow \infty$. However, since we have shown that the $\operatorname{dist}(\tilde{\mathbf{S}}, \mathbf{S})$ diverges if $r_{i, k} \rightarrow 0$ for some $i \in I$ as $k \rightarrow \infty$ regardless if the corresponding $R_{i, k}$ 
converges or diverges as $k \rightarrow \infty$, we do not need to account for this in (3.3). Note that from this it follows that $d_{T(\operatorname{IFS}(I))}\left(\tilde{\mathbf{S}}, \mathbf{S}_{k}\right)$ does not depend on the choice of $\left\{\mathbf{S}_{k}\right\}_{k \in \mathbb{N}}$.

Thus in this case, the part of the essential boundary

$$
\partial_{\mathrm{ess}}^{\mathrm{iosc}} T(\operatorname{IFS}(I)) \cup \partial_{\mathrm{ess}}^{\mathrm{niosc}} T(\operatorname{IFS}(I))
$$

is infinitely far away from $T(\operatorname{IFS}(I))$. This is clearly in a sharp contrast to the situation where the part of the essential boundary $\partial_{\mathrm{ess}}^{\mathrm{osc}} T(\operatorname{IFS}(I)) \cup \partial_{\mathrm{ess}}^{\text {nosc }} T(\operatorname{IFS}(I))$ was a finite distance from $T(\operatorname{IFS}(I))$. Note further that the part of the boundary $\partial T(\operatorname{IFS}(I)) \backslash \partial_{\text {ess }} T(\operatorname{IFS}(I))$ is infinitely far away from $T(\operatorname{IFS}(I))$ as well. This leads to the following proposition.

Proposition 3.11. If $I$ is finite with $|I|>1$ and $\tilde{\mathbf{S}}, \mathbf{S} \in T(\operatorname{IFS}(I)) \cup \partial T(\operatorname{IFS}(I))$, then the hyperbolic distance satisfies $\operatorname{dist}(\tilde{\mathbf{S}}, \mathbf{S})=\infty$ if and only if

$$
\{\tilde{\mathbf{S}}, \mathbf{S}\} \cap\left(\partial T(\operatorname{IFS}(I)) \backslash\left(\partial_{\text {ess }}^{\text {osc }} T(\operatorname{IFS}(I)) \cup \partial_{\text {ess }}^{\text {nosc }} T(\operatorname{IFS}(I))\right)\right) \neq \emptyset
$$

We fix a phase space $X$. Then we immediately have the following corollary to Proposition 3.11

Corollary 3.12. Assume that the phase space $X$ is star-shaped. If $I$ is finite and $|I|>1$, then $T(\operatorname{IFS}(X, I)) \cup \partial_{\text {ess }}^{\text {osc }} T(\operatorname{IFS}(X, I)) \cup \partial_{\text {ess }}^{\text {nosc }} T(\operatorname{IFS}(X, I))$ is complete with respect to the hyperbolic distance $\operatorname{dist}(\tilde{\mathbf{S}}, \mathbf{S})$ (which is, in this case, a metric on $\left.T(\operatorname{IFS}(X, I)) \cup \partial_{\text {ess }}^{\text {osc }} T(\operatorname{IFS}(X, I)) \cup \partial_{\text {ess }}^{\text {nosc }} T(\operatorname{IFS}(X, I))\right)$.

Secondly, we consider

$$
d_{e}\left(S_{1}, S_{2}\right):=d_{\operatorname{man}}\left(\left(a_{1}, r_{1}\right),\left(a_{2}, r_{2}\right)\right)+\min \left\{r_{1}, r_{2}\right\} d_{O(d)}\left(R_{1}, R_{2}\right) .
$$

It is easy to verify that (3.4) defines a metric on the space $\mathbb{R}^{d} \times(0,1) \times O(d)$ and hence (3.2) with $d:=d_{e}$ defines a metric on the space $T(\operatorname{IFS}(I))$ which we call the Euclidean metric $d_{T(\operatorname{IFS}(I))}$. We only need to verify the triangle inequality since the rest of the properties required by a metric clearly hold. First assume that $\min \left\{r_{1}, r_{2}\right\} \leq r_{3}$. We then have

$$
\begin{aligned}
d_{e}\left(S_{1}, S_{2}\right)= & d_{\operatorname{man}}\left(\left(a_{1}, r_{1}\right),\left(a_{2}, r_{2}\right)\right)+\min \left\{r_{1}, r_{2}\right\} d_{O(d)}\left(R_{1}, R_{2}\right) \\
\leq & d_{\operatorname{man}}\left(\left(a_{1}, r_{1}\right),\left(a_{3}, r_{3}\right)\right)+d_{\operatorname{man}}\left(\left(a_{3}, r_{3}\right),\left(a_{2}, r_{2}\right)\right) \\
& \quad+\min \left\{r_{1}, r_{2}\right\}\left(d_{O(d)}\left(R_{1}, R_{3}\right)+d_{O(d)}\left(R_{3}, R_{2}\right)\right) \\
\leq & d_{\operatorname{man}}\left(\left(a_{1}, r_{1}\right),\left(a_{3}, r_{3}\right)\right)+d_{\operatorname{man}}\left(\left(a_{3}, r_{3}\right),\left(a_{2}, r_{2}\right)\right) \\
& \quad+r_{1} d_{O(d)}\left(R_{1}, R_{3}\right)+r_{2} d_{O(d)}\left(R_{3}, R_{2}\right) \\
= & d_{\operatorname{man}}\left(\left(a_{1}, r_{1}\right),\left(a_{3}, r_{3}\right)\right)+d_{\operatorname{man}}\left(\left(a_{3}, r_{3}\right),\left(a_{2}, r_{2}\right)\right) \\
& \quad+\min \left\{r_{1}, r_{3}\right\} d_{O(d)}\left(R_{1}, R_{3}\right)+\min \left\{r_{2}, r_{3}\right\} d_{O(d)}\left(R_{3}, R_{2}\right) \\
= & d_{e}\left(S_{1}, S_{3}\right)+d_{e}\left(S_{3}, S_{2}\right) .
\end{aligned}
$$


Now suppose that $\min \left\{r_{1}, r_{2}\right\}>r_{3}$. Without loss of generality we can assume that $r_{3}<r_{2} \leq r_{1}$. Let $a_{i}:=\left(a_{1}^{i}, \ldots, a_{d}^{i}\right)$ for $i=\{1,2,3\}$. Now consider

$$
\begin{aligned}
d_{e}\left(S_{1}, S_{2}\right)= & d_{\operatorname{man}}\left(\left(a_{1}, r_{1}\right),\left(a_{2}, r_{2}\right)\right)+r_{2} d_{O(d)}\left(R_{1}, R_{2}\right) \\
= & d_{\operatorname{man}}\left(\left(a_{1}, r_{1}\right),\left(a_{2}, r_{2}\right)\right)+\left(r_{2}-r_{3}\right) d_{O(d)}\left(R_{1}, R_{2}\right)+r_{3} d_{O(d)}\left(R_{1}, R_{2}\right) \\
\leq & d_{\operatorname{man}}\left(\left(a_{1}, r_{1}\right),\left(a_{2}, r_{2}\right)\right)+\left(r_{2}-r_{3}\right)+r_{3} d_{O(d)}\left(R_{1}, R_{2}\right) \\
= & \sum_{i=1}^{d}\left|a_{i}^{1}-a_{i}^{2}\right|+\left(r_{1}-r_{2}\right)+\left(r_{2}-r_{3}\right)+r_{3} d_{O(d)}\left(R_{1}, R_{2}\right) \\
\leq & \sum_{i=1}^{d}\left|a_{i}^{1}-a_{i}^{2}\right|+\left(r_{1}-r_{3}\right)+\left(r_{2}-r_{3}\right)+r_{3} d_{O(d)}\left(R_{1}, R_{2}\right) \\
\leq & \sum_{i=1}^{d}\left|a_{i}^{1}-a_{i}^{3}\right| \\
& \quad+\sum_{i=1}^{d}\left|a_{i}^{3}-a_{i}^{2}\right|+\left(r_{1}-r_{3}\right)+\left(r_{2}-r_{3}\right)+r_{3} d_{O(d)}\left(R_{1}, R_{2}\right) \\
\leq & \sum_{i=1}^{d}\left|a_{i}^{1}-a_{i}^{3}\right|+\sum_{i=1}^{d}\left|a_{i}^{3}-a_{i}^{2}\right|+\left(r_{1}-r_{3}\right)+\left(r_{2}-r_{3}\right) \\
& +r_{3}\left(d_{O(d)}\left(R_{1}, R_{3}\right)+d_{O(d)}\left(R_{3}, R_{2}\right)\right) \\
= & d_{e}\left(S_{1}, S_{3}\right)+d_{e}\left(S_{3}, S_{2}\right) .
\end{aligned}
$$

Hence, in the case when $d:=d_{e}$, all parts of the essential boundary $\partial_{\text {ess }} T(\operatorname{IFS}(I))$ are a finite distance from $T(\operatorname{IFS}(I))$.

Note that if $r_{i, k} \rightarrow 0$ for some $i \in I$ as $k \rightarrow \infty$ and if the corresponding $R_{i, k}$ do not converge as $k \rightarrow \infty$ the metric (3.4) is still well defined since in this case we have $\min \left\{\tilde{r}_{i}, r_{i, k}\right\} \rightarrow 0$ and consequently $\min \left\{\tilde{r}_{i}, r_{i, k}\right\} d_{O(d)}\left(\tilde{R}_{i}, R_{i, k}\right) \rightarrow 0$ as $k \rightarrow \infty$.

Also, note that the part of the boundary $\partial T(\operatorname{IFS}(I)) \backslash \partial_{\text {ess }} T(\operatorname{IFS}(I))$ is infinitely far away from $T(\operatorname{IFS}(I))$ with respect to this metric. Hence, in this case $T(\operatorname{IFS}(I)) \cup \partial_{\text {ess }} T(\operatorname{IFS}(I))$ is complete.

Until now we only introduced the metrics on $T(\operatorname{IFS}(I))$ in the case of the index set $I$ being finite. We will now introduce the metrics on $T(\operatorname{IFS}(I))$ when $I$ is infinite. Clearly, in the case of $I$ being infinite, the sum in (3.2) may not converge. To take this into account, Roy and Urbanski [RU05] introduced the $\rho_{\infty}$-metric defined by

$$
\rho_{\infty}(\tilde{\mathbf{S}}, \mathbf{S}):=\sum_{i=1}^{\infty} 2^{-i} \min \left\{1,\left\|\tilde{S}_{i}-S_{i}\right\|_{X}+\left\|\tilde{S}_{i}^{\prime}-S_{i}^{\prime}\right\|_{X}\right\} .
$$

Following this approach we could modify the sum in (3.2) to

$$
\sum_{i \in I} 2^{-i} \min \left\{1, d\left(\tilde{S}_{i}, S_{i}\right)\right\}
$$

However, this would have the following disadvantage. The weights $2^{-i}$ in combination with the minimum would allow the distance $d\left(\tilde{S}_{i}, S_{i}\right)$ to be extremely large for large enough $i$ without having a big influence on (3.5) (see the example following Lemma 5.3 in [RU05]). This is obviously a disadvantage if one wants to study 
convergence of a family of IFS. To avoid such problems we introduce the following metric. For $\tilde{\mathbf{S}}, \mathbf{S} \in T(\operatorname{IFS}(I))$ we define an extended metric $d_{\mathrm{ex}}, T(\operatorname{IFS}(I))$ on the space $T(\operatorname{IFS}(I))$ by

$$
d_{\mathrm{ex}, T(\operatorname{IFS}(I))}(\tilde{\mathbf{S}}, \mathbf{S}):=\sum_{i \in I}\left(d\left(\tilde{S}_{i}, S_{i}\right)\right) .
$$

We define a metric $d_{T(\operatorname{IFS}(I))}$ on the space $T(\operatorname{IFS}(I))$ when $I$ is infinite by

$$
d_{T(\operatorname{IFS}(I))}(\tilde{\mathbf{S}}, \mathbf{S}):=\frac{d_{\mathrm{ex}, T(\operatorname{IFS}(I))}(\tilde{\mathbf{S}}, \mathbf{S})}{1+d_{\mathrm{ex}, T(\operatorname{IFS}(I))}(\tilde{\mathbf{S}}, \mathbf{S})} .
$$

Remark 3.13. Note that when $I$ is infinite we do not have an analogous statement to Proposition 3.11, since $d_{T(\operatorname{IFS}(I))}$ is always less than or equal to 1 .

\section{TEICHMÜLLER SPACE FOR CONFORMAL ITERATED FUNCTION SYSTEMS}

Following the approach from Section 3, in this section we will introduce the notion of Teichmüller space for CIFS. Thus for any fixed (finite or infinite) alphabet $I$ and for any fixed phase space $X$, let $\operatorname{CIFS}(X, I)$ denote the space of all conformal iterated function systems with phase space $X$ and alphabet $I$ and let $\operatorname{ICIFS}(X, I)$ denote the space of all inhomogeneous conformal iterated function systems with phase space $X$ and alphabet $I$. For CIFS we define the Teichmüller space $T(\operatorname{CIFS}(X, I))$ by

$$
T(\operatorname{CIFS}(X, I)):=\{\mathbf{S} \in \operatorname{CIFS}(X, I) \mid \mathbf{S} \text { satisfies } \operatorname{SSC}\} .
$$

Recall from the introduction that for $d \geq 3$, conformal maps between domains in $\mathbb{R}^{d}$ are of the form $S_{i}(x)=r_{i} R_{i} i_{c_{i}, s_{i}}(x)+a_{i}$ for $i \in I$ and where $r_{i}>0, a_{i} \in \mathbb{R}^{d}$, $R_{i} \in O(d)$ and $i_{c_{i}, s_{i}}$ is either the identity or the inversion with respect to the sphere centered at a point $c_{i}$ and with radius $s_{i}$. If $i_{c_{i}, s_{i}}$ is an inversion with respect to a sphere, we can rewrite the conformal map $S_{i}$ as

$$
S_{i}(x)=\frac{r_{i} s_{i}^{2} R_{i}\left(x-c_{i}\right)}{\left\|x-c_{i}\right\|^{2}}+r_{i} R_{i}\left(c_{i}\right)+a_{i} .
$$

Without loss of generality we can assume that $0 \in X$ and therefore $c_{i} \neq 0$ for all $i \in I$. It is easily seen that the representation in (4.1) is unique. Also note that the set of $\left\{a_{i}\right\}_{i \in I}$ is bounded since the images of $X$ under $\mathbf{S}$ have to be a subset of the bounded set $X$. This is already the first difference to the case of IFS discussed in Section 3 .

Fix $i \in I$ and let $S_{i, k}(x)=\frac{r_{i} s_{i, k}^{2} R_{i}\left(x-c_{i, k}\right)}{\left\|x-c_{i, k}\right\|^{2}}+r_{i} R_{i}\left(c_{i, k}\right)+a_{i}$ with $\left\|c_{i, k}\right\| \rightarrow \infty$ as $k \rightarrow \infty$. We will now show that the conformal map $S_{i, k}$ tends to the similarity map $P_{i}(x)=r_{i} R_{i}(x)+a_{i}$ when $\left\|c_{i, k}\right\| \rightarrow \infty$ as $k \rightarrow \infty$. Write $S_{i, k}$ as $S_{i, k}=P_{i} \circ i_{c_{i, k}, s_{i, k}}$. Then $S_{i, k}(X) \subset X$ implies that $i_{c_{i, k}, s_{i, k}}(X) \subset P_{i}^{-1}(X)$. If we change $c_{i, k}$ we also need to change $s_{i, k}$ in such a way that $\left|\left\|c_{i, k}\right\|-s_{i, k}\right|$ is bounded from above for $i_{c_{i, k}, s_{i, k}}(X) \subset P_{i}^{-1}(X)$ to hold. Hence it is easily seen that $\frac{s_{i, k}^{2}}{\left\|x-c_{i, k}\right\|^{2}} \rightarrow 1$ with $\left\|c_{i, k}\right\| \rightarrow \infty$ as $k \rightarrow \infty$. Therefore $S_{i, k} \rightarrow P_{i}$ with $\left\|c_{i, k}\right\| \rightarrow \infty$ as $k \rightarrow \infty$.

We want to study the boundary $\partial T(\operatorname{CIFS}(X, I))$ of $T(\operatorname{CIFS}(X, I))$. First we introduce the notion of the essential boundary $\partial_{\mathrm{ess}} T(\operatorname{CIFS}(X, I))$ of $T(\operatorname{CIFS}(X, I))$ as we did in Section 3. Since the set of $\left\{a_{i}\right\}_{i \in I}$ is bounded, we have to do this differently to the case of IFS. Therefore we first clarify what are the pathological cases for $\partial T(\operatorname{CIFS}(X, I))$. We call a system $\mathbf{S}=\left\{S_{i}\right\}_{i \in I}$ pathological if we have that $1 \leq$ 
$\left|S_{i}^{\prime}(x)\right|$ for some $x \in X$ and for some $i \in I$. We exclude all such pathological systems from what we call the essential boundary $\partial_{\text {ess }} T(\operatorname{CIFS}(X, I))$ of $T(\operatorname{CIFS}(X, I))$. Let $\left\{\mathbf{S}_{k}\right\}_{k \in \mathbb{N}}=\left\{\left\{S_{i, k}\right\}_{i \in I}\right\}_{k \in \mathbb{N}}$ be a sequence of CIFS in $T(\operatorname{CIFS}(X, I))$ which converges pointwise to $\mathbf{S}=\left\{S_{i}\right\}_{i \in I}$ in $\partial T(\operatorname{CIFS}(X, I))$. Note that $\left\{\mathbf{S}_{k}\right\}_{k \in \mathbb{N}} \in T(\operatorname{CIFS}(X, I))$ may converge to a system $\mathbf{S} \in \partial T(\operatorname{CIFS}(X, I))$ which is not conformal in the sense that there does not exist an open connected set $V$ with $X \subset V \subset \mathbb{R}^{d}$ such that all maps $S_{i}, i \in I$, extend to $C^{1}$ conformal diffeomorphisms of $V$ into $V$. We exclude such cases from $\partial_{\text {ess }} T(\operatorname{CIFS}(X, I))$ as well.

The next theorem provides a complete description of the essential boundary $\partial_{\text {ess }} T(\operatorname{CIFS}(X, I))$.

Theorem 4.1. Assume that the phase space $X$ is star-shaped. Then for $d \geq 3$ we have,

(1) $\{\mathbf{S} \in C I F S(X, I) \mid \mathbf{S}$ does not satisfy $S S C\} \subset \partial_{\text {ess }} T(\operatorname{CIFS}(X, I))$,

(2) $\partial_{\text {ess }} T(\operatorname{CIFS}(X, I)) \backslash\{\mathbf{S} \in C I F S(X, I) \mid \mathbf{S}$ does not satisfy $S S C\}$

$$
\subset\left\{\mathbf{S}_{C} \in \operatorname{ICIFS}(X, J) \mid J \subsetneq I\right\} \text {. }
$$

Proof. The proof is very similar to the proof of Theorem 3.4 and is therefore omitted.

Remark 4.2. Since in the case of CIFS the starting set $X$ is fixed and therefore cannot collapse to a point we do not have the situation that $L\left(\mathbf{S}_{k}\right) \rightarrow\{p\}$ as $k \rightarrow \infty$ and $p \in \mathbb{R}^{d}$ as in Theorem 3.4.

For ease of exposition we write

$$
\partial_{\text {ess }} T(\operatorname{CIFS}(X, I))=\partial_{\mathrm{ess}}^{\text {osc }} T(\operatorname{CIFS}(X, I)) \cup \partial_{\mathrm{ess}}^{\mathrm{iosc}} T(\operatorname{CIFS}(X, I)),
$$

where $\partial_{\mathrm{ess}}^{\mathrm{osc}} T(\operatorname{CIFS}(X, I)):=\{\mathbf{S} \in \operatorname{CIFS}(X, I) \mid \mathbf{S}$ does not satisfy $\mathrm{SSC}\}$ and $\partial_{\mathrm{ess}}^{\mathrm{iosc}} T(\operatorname{CIFS}(X, I)):=\left\{\mathbf{S}_{C} \in \operatorname{ICIFS}(X, J) \mid J \subsetneq I\right\} \cap \partial_{\mathrm{ess}} T(\operatorname{CIFS}(X, I))$.

4.1. Metrics on $T(\operatorname{CIFS}(X, I))$ for $d \geq 3$. Recall that, for $d \geq 3$ we can represent each conformal map $S_{i}$ by a point $\left(a_{i}, r_{i}, R_{i}, c_{i}, s_{i}\right)$ in the space $\mathbb{R}^{d} \times(0,1) \times O(d) \times$ $S^{d} \times(0, \infty] \subset \mathbb{R}^{d} \times(0,1) \times O(d) \times S^{d} \times S^{1}$, where $S^{d}=\left\{\mathbb{R}^{d} \cup\{\infty\}\right\}$ is a $d$-dimensional Riemann sphere. Clearly, it is possible to equip $S^{d}$ with a metric $d_{S^{d}}$ such that $\operatorname{diam}\left(S^{d}\right) \leq \frac{1}{3}$, i.e. $d_{S^{d}}(a, b) \leq \frac{1}{3}$ for all $a, b \in S^{d}$ and in this section we equip $O(d)$ with a metric $d_{O(d)}$ such that $\operatorname{diam}(O(d)) \leq \frac{1}{3}$. Following the approach from Section 3.1 we now define the distance between two conformal maps $S_{1}$ and $S_{2}$ in the following two ways

$$
\begin{aligned}
d_{h}\left(S_{1}, S_{2}\right):=d_{\mathrm{hyp}}\left(\left(a_{1}, r_{1}\right),\left(a_{2}, r_{2}\right)\right)+d_{O(d)}( & \left.R_{1}, R_{2}\right) \\
& +d_{S^{d}}\left(c_{1}, c_{2}\right)+d_{S^{1}}\left(s_{1}, s_{2}\right),
\end{aligned}
$$

and

$$
\begin{aligned}
d_{e}\left(S_{1}, S_{2}\right):= & d_{\operatorname{man}}\left(\left(a_{1}, r_{1}\right),\left(a_{2}, r_{2}\right)\right) \\
& +\min \left\{r_{1}, r_{2}\right\}\left(d_{O(d)}\left(R_{1}, R_{2}\right)+d_{S^{d}}\left(c_{1}, c_{2}\right)+d_{S^{1}}\left(s_{1}, s_{2}\right)\right) .
\end{aligned}
$$

As before both (4.2) and (4.3) define a metric on the space $\mathbb{R}^{d} \times(0,1) \times O(d) \times S^{d} \times S^{1}$ and are natural generalizations of (3.3) and (3.4). Following the approach for IFS, let $\tilde{\mathbf{S}}=\left\{\tilde{S}_{i}\right\}_{i \in I}$ be any CIFS in $T(\operatorname{CIFS}(X, I))$ and let $\left\{\mathbf{S}_{k}\right\}_{k \in \mathbb{N}}=\left\{\left\{S_{i, k}\right\}_{i \in I}\right\}_{k \in \mathbb{N}}$ be a sequence of CIFS in $T(\operatorname{CIFS}(X, I))$ which converges pointwise to $\mathbf{S}=\left\{S_{i}\right\}_{i \in I}$ 
in $\partial_{\text {ess }} T(\operatorname{CIFS}(X, I))$. For a finite alphabet $I$ we define the distance between $\tilde{\mathbf{S}}$ and $\mathbf{S}$ by

$$
\operatorname{dist}(\tilde{\mathbf{S}}, \mathbf{S}):=\lim _{k \rightarrow \infty} d_{T(\operatorname{CIFS}(X, I))}\left(\tilde{\mathbf{S}}, \mathbf{S}_{k}\right),
$$

where for all $k \in \mathbb{N}$ we define the distance $d_{T(\operatorname{CIFS}(X, I))}$ between $\tilde{\mathbf{S}}$ and $\mathbf{S}_{k}$ by

$$
d_{T(\operatorname{CIFS}(X, I))}\left(\tilde{\mathbf{S}}, \mathbf{S}_{k}\right):=\sum_{i \in I}\left(d\left(\tilde{S}_{i}, S_{i, k}\right)\right) .
$$

If at least one of the conformal maps $S_{i, k}$ in the sequence of CIFS $\left\{\mathbf{S}_{k}\right\}_{k \in \mathbb{N}}$ in $T(\operatorname{CIFS}(X, I))$ converges pointwise to a condensation transformation, i.e. $\left|S_{i, k}^{\prime}\right| \rightarrow 0$ for some $i \in I$ as $k \rightarrow \infty$, this means that the corresponding $r_{i, k} \rightarrow 0$ and hence the corresponding $R_{i, k}$ and $i_{c_{i, k}}$ do not need to converge as $k \rightarrow \infty$. Exactly the same calculations as in Section 3.1 show that $\operatorname{dist}(\tilde{\mathbf{S}}, \mathbf{S})$ with $d:=d_{h}$ diverges if $r_{i, k} \rightarrow 0$ for some $i \in I$ as $k \rightarrow \infty$ regardless if the corresponding $R_{i, k}$ and $i_{c_{i, k}}$ converge or diverge as $k \rightarrow \infty$ and thus again we do not need to account for this in (4.2). The metric (4.3) is still well defined since in this case we again have $\min \left\{\tilde{r}_{i}, r_{i, k}\right\} \rightarrow 0$ and consequently $\min \left\{\tilde{r}_{i}, r_{i, k}\right\}\left(d_{O(d)}\left(\tilde{R}_{i}, R_{i, k}\right)+d_{S^{d}}\left(\tilde{c}_{i}, c_{i, k}\right)+d_{S^{1}}\left(\tilde{s}_{i}, s_{i, k}\right)\right) \rightarrow 0$ as $k \rightarrow \infty$. This leads to the following proposition.

Proposition 4.3. If $I$ is finite and $\tilde{\mathbf{S}}, \mathbf{S} \in T(\operatorname{CIFS}(X, I)) \cup \partial_{\text {ess }} T(\operatorname{CIFS}(X, I))$, then the hyperbolic distance satisfies $\operatorname{dist}(\tilde{\mathbf{S}}, \mathbf{S})=\infty$ if and only if

$$
\{\tilde{\mathbf{S}}, \mathbf{S}\} \cap \partial_{\text {ess }}^{i o s c} T(\operatorname{CIFS}(X, I)) \neq \emptyset .
$$

Remark 4.4. The hyperbolic distance $\operatorname{dist}(\tilde{\mathbf{S}}, \mathbf{S})$ is a metric on $T(\operatorname{CIFS}(X, I)) \cup$ $\partial_{\text {ess }}^{\text {osc }} T(\operatorname{CIFS}(X, I))$. However, since a sequence of CIFS might converge to a system which is no longer conformal, we do not have an analog of Corollary 3.12 for conformal systems.

We will now introduce metrics on $T(\operatorname{CIFS}(X, I))$ when $I$ is infinite. As in Section 3.1, the sum in (4.5) may not converge. Hence in this case for $\tilde{\mathbf{S}}, \mathbf{S} \in T(\operatorname{CIFS}(X, I))$ we define an extended metric $d_{\mathrm{ex}}, T(\operatorname{CIFS}(X, I))$ on the space $T(\operatorname{CIFS}(X, I))$ by

$$
d_{\mathrm{ex}, T(\operatorname{CIFS}(X, I))}(\tilde{\mathbf{S}}, \mathbf{S}):=\sum_{i \in I}\left(d\left(\tilde{S}_{i}, S_{i}\right)\right) .
$$

We define a metric $d_{T(\operatorname{CIFS}(X, I))}$ on the space $T(\operatorname{CIFS}(X, I))$ when $I$ is infinite by

$$
d_{T(\operatorname{CIFS}(X, I))}(\tilde{\mathbf{S}}, \mathbf{S}):=\frac{d_{\mathrm{ex}, T(\operatorname{CIFS}(X, I))}(\tilde{\mathbf{S}}, \mathbf{S})}{1+d_{\mathrm{ex}, T(\operatorname{CIFS}(X, I))}(\tilde{\mathbf{S}}, \mathbf{S})} .
$$

Remark 4.5. Note that when $I$ is infinite we do not have an analogous statement to Proposition 4.3, since $d_{T(\operatorname{CIFS}(X, I))}$ is always less than or equal to 1 .

\section{Convergence in the $\lambda$-Topology vs. Convergence}

$$
\text { WITH RESPECT TO } d_{T(\operatorname{IFS}(X, I))}
$$

Throughout this section we assume that the alphabet $I$ is countably infinite. In RU05] Roy and Urbaski introduced the notion of $\lambda$-topology. In this section we will show that convergence with respect to the hyperbolic metric $d_{T(\operatorname{IFS}(X, I))}$ implies convergence in the $\lambda$-topology. First we recall the definition of the $\lambda$-topology. Given a sequence of CIFS $\left\{\mathbf{S}_{k}\right\}_{k \in \mathbb{N}}=\left\{\left\{S_{i, k}\right\}_{i \in I}\right\}_{k \in \mathbb{N}}$ and $\mathbf{S}=\left\{S_{i}\right\}_{i \in I}$ sharing the 
same alphabet $I$ and the same phase space $X$ we say that $\left\{\mathbf{S}_{k}\right\}_{k \in \mathbb{N}}$ converges to $\mathbf{S}$ in the $\lambda$-topology if $\mathbf{S}_{k} \rightarrow \mathbf{S}$ in the $\rho_{\infty}$-metric given by

$$
\rho_{\infty}\left(\mathbf{S}_{k}, \mathbf{S}\right):=\sum_{i=1}^{\infty} 2^{-i} \min \left\{1,\left\|S_{i, k}-S_{i}\right\|_{X}+\left\|S_{i}^{\prime}-S_{i, k}^{\prime}\right\|_{X}\right\}
$$

and if there exist $C>0$ and $M \in \mathbb{N}$ such that

$$
\left|\log \left\|S_{i}^{\prime}\right\|_{X}-\log \left\|S_{i, k}^{\prime}\right\|_{X}\right| \leq C
$$

for all $i \in I$ and all $k \geq M$. Here, $\|\cdot\|_{X}$ denotes the supremum norm taken over $X$, that is $\left\|S_{i}\right\|_{X}:=\sup _{x \in X}\left\|S_{i}(x)\right\|$.

Theorem 5.1. If $\mathbf{S}_{k}, \mathbf{S} \in T(\operatorname{IFS}(X, I))$, and $\mathbf{S}_{k} \rightarrow \mathbf{S}$ in the hyperbolic metric $d_{T(I F S(X, I))}$, then $\mathbf{S}_{k} \rightarrow \mathbf{S}$ in the $\lambda$-topology.

Proof. Observe that if $\mathbf{S}_{k} \rightarrow \mathbf{S}$ as $k \rightarrow \infty$ in the hyperbolic metric $d_{T(\operatorname{IFS}(X, I))}$, then $d_{\mathrm{ex}, T(\operatorname{IFS}(X, I))}\left(\mathbf{S}, \mathbf{S}_{k}\right)=\sum_{i \in I}\left(d_{h}\left(S_{i}, S_{i, k}\right)\right) \rightarrow 0$. In particular, for any constant $C>0$ there exists $K \in \mathbb{N}$ such that $d_{h}\left(S_{i}, S_{i, k}\right)<C$ for all $k \geq K$ and all $i \in I$. This implies that

$$
\left|\log r_{i}-\log r_{i, k}\right| \leq d_{\text {hyp }}\left(\left(a_{i}, r_{i}\right),\left(a_{i, k}, r_{i, k}\right)\right)<C \quad \text { for all } k \geq K .
$$

Thus for all $k \geq K$ we have

$$
\left|\log \left\|S_{i}^{\prime}\right\|_{X}-\log \left\|S_{i, k}^{\prime}\right\|_{X}\right|=\left|\log r_{i}-\log r_{i, k}\right| \leq C .
$$

This completes the proof.

In RSU09 it was shown that the $\lambda$-topology is not metrizable. However, Theorem 5.1 shows that in the case of IFS we have found a good metric which implies convergence in the $\lambda$-topology. Therefore, it seems to us that this metric should be studied further which we do in the next sections.

\section{Continuity of Hausdorff dimension for IFS}

In this section we will investigate the continuity of the Hausdorff dimension function. Let $h=h_{\mathbf{S}}$ denote the Hausdorff dimension of the dynamical limit set $L_{\text {dyn }}(\mathbf{S})$. Recall that $L(\mathbf{S})=L_{\text {dyn }}(\mathbf{S})$ if $I$ is finite. It is well known (see Hut81]) that if $\mathbf{S}$ satisfies OSC and that if $I$ is finite, then the Hausdorff dimension $h_{\mathbf{S}}$ of the dynamical limit set $L_{\mathrm{dyn}}(\mathbf{S})$ is given by

$$
\sum_{i \in I} r_{i}^{h_{\mathrm{S}}}=1
$$

Theorem 6.1. If I is finite, then the Hausdorff dimension function

$$
\begin{gathered}
h: T(\operatorname{IFS}(I)) \cup\left(\partial_{\text {ess }} T(\operatorname{IFS}(I)) \backslash\left(\partial_{\text {ess }}^{\text {nosc }} T(\operatorname{IFS}(I)) \cup \partial_{\text {ess }}^{\text {niosc }} T(\operatorname{IFS}(I))\right)\right) \rightarrow[0, \infty), \\
\mathbf{S} \longmapsto h_{\mathbf{S}},
\end{gathered}
$$

is continuous with respect to the Euclidean metric $d_{T(I F S(I))}$.

Proof. Let $\left\{\mathbf{S}_{k}\right\}_{k \in \mathbb{N}}=\left\{\left\{S_{i, k}\right\}_{i \in I}\right\}_{k \in \mathbb{N}}$ be a sequence of IFS in $T(\operatorname{IFS}(I))$ converging pointwise to $\mathbf{S}=\left\{S_{i}\right\}_{i \in I}$ in $\partial_{\mathrm{ess}} T(\operatorname{IFS}(I)) \backslash\left(\partial_{\mathrm{ess}}^{\text {nosc }} T(\operatorname{IFS}(I)) \cup \partial_{\mathrm{ess}}^{\text {niosc }} T(\operatorname{IFS}(I))\right)$. Let $\left\{h_{\mathbf{S}_{k}}\right\}_{k \in \mathbb{N}}$ be the corresponding sequence of the Hausdorff dimensions of the dynamical limit sets $L_{\mathrm{dyn}}\left(\mathbf{S}_{k}\right)$. 
Case 1. $\mathbf{S} \in \partial_{\mathrm{ess}}^{\mathrm{osc}} T(\operatorname{IFS}(I))$.

Since $r_{i, k} \rightarrow r_{i}$ when $k \rightarrow \infty$ we clearly have $h_{\mathbf{S}_{k}} \rightarrow h_{\mathbf{S}}$ as $k \rightarrow \infty$. Thus the Hausdorff dimension function $h: T(\operatorname{IFS}(I)) \cup \partial_{\mathrm{ess}}^{\mathrm{osc}} T(\operatorname{IFS}(I)) \rightarrow[0, \infty), \mathbf{S} \mapsto h_{\mathbf{S}}$, is clearly continuous.

Case 2. $\mathbf{S} \in \partial_{\text {ess }}^{\text {iosc }} T(\operatorname{IFS}(I))$. We can write $\mathbf{S}_{k}$ as $\mathbf{S}_{k}=\left\{S_{i, k}\right\}_{i \in I_{1}} \cup\left\{S_{i, k}\right\}_{i \in I_{2}}$, where $I_{1}:=\left\{i \in I \mid r_{i, k} \rightarrow r_{i} \neq 0\right.$ as $\left.k \rightarrow \infty\right\}$ and $I_{2}:=\left\{i \in I \mid r_{i, k} \rightarrow 0\right.$ as $\left.k \rightarrow \infty\right\}$ and $I=I_{1} \cup I_{2}$. Therefore we can write $\mathbf{S}$ as $\mathbf{S}=\mathbf{S}_{1} \cup \mathbf{S}_{2}$ where $\mathbf{S}_{1}=\left\{S_{i}\right\}_{i \in I_{1}}$ and $\mathbf{S}_{2}=\left\{a_{i}\right\}_{i \in I_{2}}$. It now follows from Lemma 2.5 that the Hausdorff dimension $h_{\mathbf{S}}$ of the dynamical limit set $L_{\mathrm{dyn}}(\mathbf{S})$ is given by

$$
h_{\mathbf{S}}=\max \left(h_{\mathbf{S}_{1}}, \operatorname{dim}_{\mathrm{H}}\left(\left\{a_{i}\right\}_{i \in I_{2}}\right)\right)=h_{\mathbf{S}_{1}},
$$

where

$$
\sum_{i \in I_{1}} r_{i}^{h_{\mathbf{s}_{1}}}=1
$$

Since $r_{i, k} \rightarrow r_{i}$ for $i \in I_{1}$ when $k \rightarrow \infty$ we have $h_{\mathbf{S}_{k}} \rightarrow h_{\mathbf{S}_{1}}$ as $k \rightarrow \infty$. Thus the Hausdorff dimension function $h: T(\operatorname{IFS}(I)) \cup \partial_{\mathrm{ess}}^{\mathrm{iosc}} T(\operatorname{IFS}(I)) \rightarrow[0, \infty), \mathbf{S} \mapsto h_{\mathbf{S}}$, is clearly continuous.

Remark 6.2. One can easily see that the Hausdorff dimension function does not extend continuously to $\partial_{\mathrm{ess}}^{\text {nosc }} T(\operatorname{IFS}(I)) \cup \partial_{\mathrm{ess}}^{\text {niosc }} T(\operatorname{IFS}(I))$ by considering IFSs from Example 3.9. The Hausdorff dimension of the limit set of these IFS is given by $\left(\frac{1}{3}\right)^{h}+r^{h}=1$ for all parameters $r \in\left(0, \frac{2}{3}\right)$ and $a \in \mathbb{R} \backslash 0$, i.e. when OSC is satisfied. Clearly $h \neq 0$ in this case. However on the part of the boundary consisting of IFS with $S_{2}(x)=r x, a=0$, namely on $\partial_{\mathrm{ess}}^{\text {nosc }} T(\operatorname{IFS}(I))$, the Hausdorff dimension is 0 and $h \nrightarrow 0$ as $a \rightarrow 0$.

Similarly, one can see that the Hausdorff dimension function does not extend continuously to $\partial_{\mathrm{ess}}^{\text {niosc }} T(\operatorname{IFS}(I))$. Namely, we slightly change the above setting and consider an IFS with three generators and deform two of them. Hence, let $S_{1}$ and $S_{2}$ be as in Example 3.9 and $S_{3}=\tilde{r} x+\tilde{a}, \tilde{r} \in(0,1), \tilde{a} \in \mathbb{R}$. In this case the Hausdorff dimension is given by $\left(\frac{1}{3}\right)^{h}+r^{h}+\tilde{r}^{h}=1$ for all parameters $r, a, \tilde{r}, \tilde{a}$ for which OSC is satisfied (we do not specify these parameters explicitly as it is not essential in this case). However when $a=\tilde{a}=0$ and additionally $\tilde{r}=0$ and $r \neq 0$, namely on $\partial_{\mathrm{ess}}^{\text {niosc }} T(\operatorname{IFS}(I))$, the Hausdorff dimension is 0 and $h \nrightarrow 0$ as $\tilde{r}, \tilde{a}, a \rightarrow 0$.

Remark 6.3. Theorem 6.1 holds for the packing dimension function as well.

\section{Metrics on $T(\operatorname{CIFS}(X, I))$ For all Dimensions}

In Section 4.1 we introduced metrics for the case $d \geq 3$. In this section we want to introduce other metrics which can be used in all dimensions and compare them. Recall that for $\tilde{\mathbf{S}}, \mathbf{S} \in T(\operatorname{CIFS}(X, I))$ with $I$ being infinite we define an extended metric $d_{\mathrm{ex}}, T(\operatorname{CIFS}(X, I))$ on the space $T(\operatorname{CIFS}(X, I))$ by

$$
d_{\mathrm{ex}, T(\operatorname{CIFS}(X, I))}(\tilde{\mathbf{S}}, \mathbf{S})=\sum_{i \in I}\left(d\left(\tilde{S}_{i}, S_{i}\right)\right) .
$$

Now let $d\left(\tilde{S}_{i}, S_{i}\right):=d_{\lambda}\left(\tilde{S}_{i}, S_{i}\right)$, where we define $d_{\lambda}$ by

$$
d_{\lambda}\left(\tilde{S}_{i}, S_{i}\right):=\left|\log \left\|\tilde{S}_{i}^{\prime}\right\|_{X}-\log \left\|S_{i}^{\prime}\right\|_{X}\right|+\left\|\tilde{S}_{i}-S_{i}\right\|_{X}+\left\|\tilde{S}_{i}^{\prime}-S_{i}{ }^{\prime}\right\|_{X} .
$$

We will call a metric $d_{T(\operatorname{CIFS}(X, I))}$ the $\lambda$-metric $d_{T(\operatorname{CIFS}(X, I))}$ if $d:=d_{\lambda}$. 
To state our next results we need the following definition.

Definition 7.1 (Strong Convergence Property). Let $\tilde{\mathbf{S}}, \mathbf{S} \in \mathrm{T}((\mathrm{C}) \operatorname{IFS}(X, I))$ with $I$ being infinite. A metric $d_{\mathrm{ex}}, T((\mathrm{C}) \operatorname{IFS}(X, I))$ is said to satisfy the Strong Convergence Property (SCP) if $d\left(\tilde{S}_{i}, S_{i}\right) \rightarrow 0$ as $i \rightarrow \infty$ implies that $\left\|S_{i}-\tilde{S}_{i}\right\|_{X} \rightarrow 0$ as $i \rightarrow \infty$.

Note that

$$
\begin{aligned}
d_{\mathrm{H}}\left(\tilde{S}_{i}(X), S_{i}(X)\right) & =\max \left\{\sup _{x \in S_{i}(X)} \inf _{y \in \tilde{S}_{i}(X)} d(x, y), \sup _{x \in \tilde{S}_{i}(X)} \inf _{y \in S_{i}(X)} d(x, y)\right\} \\
& =\max \left\{\sup _{x \in X} \inf _{y \in X} d\left(S_{i}(x), \tilde{S}_{i}(y)\right), \sup _{y \in X} \inf _{x \in X} d\left(S_{i}(x), \tilde{S}_{i}(y)\right)\right\} \\
& \leq \max \left\{\sup _{x \in X} d\left(S_{i}(x), \tilde{S}_{i}(x)\right), \sup _{y \in X} d\left(S_{i}(y), \tilde{S}_{i}(y)\right)\right\} \\
(7.2) & =\left\|S_{i}-\tilde{S}_{i}\right\|_{X},
\end{aligned}
$$

where $d_{\mathrm{H}}$ denotes the Hausdorff distance. Hence $\left\|S_{i}-\tilde{S}_{i}\right\|_{X} \rightarrow 0$ as $i \rightarrow \infty$ implies that $d_{\mathrm{H}}\left(\tilde{S}_{i}(X), S_{i}(X)\right) \rightarrow 0$ as $i \rightarrow \infty$. We will use this observation in the next section. Note as well that $d_{T((\mathrm{C}) \operatorname{IFS}(X, I))}=\frac{d_{\mathrm{ex}, T((\mathrm{C}) \operatorname{IFS}(X, I))}}{1+d_{\mathrm{ex}, T((\mathrm{C}) \operatorname{IFS}(X, I))}}$ satisfies SCP if and only if $d_{\mathrm{ex}}, T((\mathrm{C}) \operatorname{IFS}(X, I))$ satisfies SCP. We now verify which of the metrics we have introduced satisfy SCP.

Proposition 7.2. The following metrics satisfy $S C P$ :

(1) $d_{e x, T(I F S(X, I))}$ with $d:=d_{h}$.

(2) $d_{e x, T(I F S(X, I))}$ with $d:=d_{e}$.

(3) $d_{e x, T(C I F S(X, I))}$ with $d:=d_{\lambda}$.

Proof. (1) Since $X$ is bounded, we have that

$$
\left\|R_{i}-\tilde{R}_{i}\right\|_{X} \leq C d_{O(d)}\left(R_{i}, \tilde{R}_{i}\right)
$$

where $C>0$ is some constant,

$$
\begin{aligned}
& \left\|S_{i}-\tilde{S}_{i}\right\|_{X}=\sup _{x \in X}\left\|S_{i}(x)-\tilde{S}_{i}(x)\right\| \\
& \leq \sup _{x \in X}\left\|r_{i} R_{i}(x)-\tilde{r}_{i} \tilde{R}_{i}(x)\right\|+\left\|a_{i}-\tilde{a}_{i}\right\| \\
& \leq \sup _{x \in X}\left(\left\|r_{i} R_{i}(x)-\tilde{r}_{i} R_{i}(x)\right\|+\left\|\tilde{r}_{i} R_{i}(x)-\tilde{r}_{i} \tilde{R}_{i}(x)\right\|\right)+\left\|a_{i}-\tilde{a}_{i}\right\| \\
& \leq \sup _{x \in X}\left\|r_{i} R_{i}(x)-\tilde{r}_{i} R_{i}(x)\right\|+\sup _{x \in X}\left\|\tilde{r}_{i} R_{i}(x)-\tilde{r_{i}} \tilde{R}_{i}(x)\right\|+\left\|a_{i}-\tilde{a_{i}}\right\| \\
& =\left|r_{i}-\tilde{r}_{i}\right| \sup _{x \in X}\left\|R_{i}(x)\right\|+\tilde{r}_{i} \sup _{x \in X}\left\|R_{i}(x)-\tilde{R}_{i}(x)\right\|+\left\|a_{i}-\tilde{a_{i}}\right\| \\
& =\left|r_{i}-\tilde{r_{i}}\right| \sup _{x \in X}\|x\|+\tilde{r}_{i}\left\|R_{i}-\tilde{R}_{i}\right\|_{X}+\left\|a_{i}-\tilde{a_{i}}\right\| \\
& \leq\left|\log r_{i}-\log \tilde{r}_{i}\right| \sup _{x \in X}\|x\|+\tilde{r}_{i}\left\|R_{i}-\tilde{R}_{i}\right\|_{X}+\left\|a_{i}-\tilde{a_{i}}\right\| \\
& \leq d_{\text {hyp }}\left(\left(a_{i}, r_{i}\right),\left(\tilde{a}_{i}, \tilde{r}_{i}\right)\right) \sup _{x \in X}\|x\|+\tilde{r}_{i} C d_{O(d)}\left(R_{i}, \tilde{R}_{i}\right) \\
& +d_{\text {hyp }}\left(\left(a_{i}, r_{i}\right),\left(\tilde{a_{i}}, \tilde{r}_{i}\right)\right) \\
& \rightarrow 0 \quad \text { as } i \rightarrow \infty \text {, if } d\left(\tilde{S}_{i}, S_{i}\right) \rightarrow 0 \text { as } i \rightarrow \infty \text {. }
\end{aligned}
$$


(2) First note that $d_{e}\left(\tilde{S}_{i}, S_{i}\right) \rightarrow 0$ as $i \rightarrow \infty$ implies that $\left\|a_{i}-\tilde{a}_{i}\right\| \rightarrow 0$ as $i \rightarrow \infty$. As above we have that

$$
\begin{aligned}
\left\|S_{i}-\tilde{S}_{i}\right\|_{X} & \leq\left|r_{i}-\tilde{r_{i}}\right| \sup _{x \in X}\|x\|+\tilde{r}_{i}\left\|R_{i}-\tilde{R}_{i}\right\|_{X}+\left\|a_{i}-\tilde{a}_{i}\right\| \\
& \leq d_{\operatorname{man}}\left(\left(a_{i}, r_{i}\right),\left(\tilde{a}_{i}, \tilde{r}_{i}\right)\right) \sup _{x \in X}\|x\|+\tilde{r}_{i} C d_{O(d)}\left(R_{i}, \tilde{R}_{i}\right)+\left\|a_{i}-\tilde{a}_{i}\right\| \\
& \rightarrow 0 \text { as } i \rightarrow \infty, \text { if } d\left(\tilde{S}_{i}, S_{i}\right) \rightarrow 0 \text { as } i \rightarrow \infty .
\end{aligned}
$$

(3) The desired result follows immediately from the fact that

$$
d_{\lambda}\left(\tilde{S}_{i}, S_{i}\right)=\left|\log \left\|\tilde{S}_{i}^{\prime}\right\|_{X}-\log \left\|S_{i}^{\prime}\right\|_{X}\right|+\left\|\tilde{S}_{i}-S_{i}\right\|_{X}+\left\|\tilde{S}_{i}^{\prime}-S_{i}^{\prime}\right\|_{X} \geq\left\|\tilde{S}_{i}-S_{i}\right\|_{X}
$$

Definition 7.3 (Lambda Convergence Property). Let $\left\{\mathbf{S}_{k}\right\}_{k \in \mathbb{N}}$ be a sequence of (C)IFS in $T((\mathrm{C}) \operatorname{IFS}(X, I))$ and let $\mathbf{S}$ be in $T((\mathrm{C}) \operatorname{IFS}(X, I))$. A metric $d_{T((\mathrm{C}) \operatorname{IFS}(X, I))}$ is said to satisfy the Lambda Convergence Property (LCP) if $\mathbf{S}_{k} \rightarrow \mathbf{S}$ in the metric $d_{T((\mathrm{C}) \operatorname{IFS}(X, I))}$ implies that $\mathbf{S}_{k} \rightarrow \mathbf{S}$ in the $\lambda$-topology.

We now verify which of the metrics we have introduced satisfy LCP.

Proposition 7.4. The following metrics satisfy LCP:

(1) $d_{T(I F S(X, I))}$ with $d:=d_{h}$.

(2) $d_{T(C I F S(X, I))}$ with $d:=d_{\lambda}$.

Proof. (1) The desired result follows immediately from Theorem 5.1

(2) The required result clearly follows from the definition of $d_{\lambda}$, i.e. from equation (7.1).

In RU05] the following theorem concerning the continuity of the pressure function was proven. Recall that the topological pressure $P(t)=P_{\mathbf{S}}(t), t \geq 0$, is defined as follows. For every $n \in \mathbb{N}$, set

$$
P^{n}(t):=\sum_{\mathbf{i} \in I^{n}}\left\|S_{\mathbf{i}}^{\prime}\right\|^{t} .
$$

Then

$$
P(t):=\lim _{n \rightarrow \infty} \frac{1}{n} \log P^{n}(t)=\inf _{n \in \mathbb{N}} \frac{1}{n} \log P^{n}(t) .
$$

Theorem 7.5 (see [RU05]). For every $t \geq 0$, the function

$$
\begin{aligned}
P(t): T(\operatorname{CIFS}(X, I)) \cup \partial_{e s s}^{o s c} T(\operatorname{CIFS}(X, I)) \rightarrow(-\infty, \infty], & \mathbf{S} \longmapsto P_{\mathbf{S}}(t),
\end{aligned}
$$

is continuous when $T(\operatorname{CIFS}(X, I)) \cup \partial_{\text {ess }}^{\text {osc }} T(\operatorname{CIFS}(X, I))$ is equipped with the $\lambda$ topology.

Combining Proposition 7.4 and Theorem 7.5 we have the following corollary concerning the continuity of the pressure function.

Corollary 7.6. For every $t \geq 0$, the function

$$
\begin{aligned}
P(t): T(\operatorname{CIFS}(X, I)) \cup \partial_{\text {ess }}^{\text {osc }} T(\operatorname{CIFS}(X, I)) \rightarrow(-\infty, \infty], \\
\mathbf{S} \longmapsto P_{\mathbf{S}}(t),
\end{aligned}
$$


is continuous when $T(\operatorname{CIFS}(X, I)) \cup \partial_{\text {ess }}^{\text {osc }} T(\operatorname{CIFS}(X, I))$ is equipped with a metric $d_{T(C I F S(X, I))}$ satisfying LCP.

Proposition 7.4 shows that in the case of CIFS we have found a good metric which implies convergence in the $\lambda$-topology. We will study this metric further in the next sections.

\section{Set of accumulation points for (C)IFS}

In this section we want to recall the notion of the set of accumulation points for (C)IFS and study its continuity properties.

Definition 8.1. For a (C)IFS S satisfying SSC we define the set of accumulation points $\operatorname{Acc}(\mathbf{S})$ by

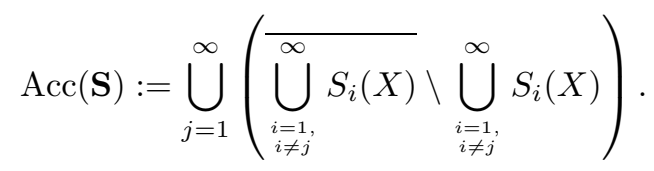

Note that the notion of $\operatorname{Acc}(\mathbf{S})$ was first introduced (and extended to (C)IFS satisfying OSC) in Hil11 and we refer there for further details. In order to prove the results in this section we need to recall the following theorem from [Hil11.

Theorem 8.2 ([Hil11]). For $\mathbf{S} \in T((C) I F S(X, I))$ with I infinite, define

$$
\mathcal{O}_{\mathbf{S}}(\operatorname{Acc}(\mathbf{S})):=\bigcup_{\mathbf{i} \in I^{*}} S_{\mathbf{i}}(\operatorname{Acc}(\mathbf{S})) \cup \operatorname{Acc}(\mathbf{S}) .
$$

We then have that

$$
L_{J}(\mathbf{S}) \subseteq \mathcal{O}_{\mathbf{S}}(\operatorname{Acc}(\mathbf{S})) \subseteq L(\mathbf{S}) .
$$

Our next theorem states conditions under which the set of accumulation points is constant.

Theorem 8.3. Let $\mathbf{S}, \mathbf{T} \in(C) \operatorname{IFS}(X, I)$ with infinite I. If $d_{T((C) I F S(X, I))}(\mathbf{S}, \mathbf{T})<$ 1 and $d_{T((C) I F S(X, I))}$ satisfies $S C P$, then we have

$$
\operatorname{Acc}(\mathbf{S})=\operatorname{Acc}(\mathbf{T}) \text {. }
$$

Proof. First note that if $d_{T((\mathrm{C}) \operatorname{IFS}(X, I))}(\mathbf{S}, \mathbf{T})<1$, then $d_{\mathrm{ex}, T((\mathrm{C}) \operatorname{IFS}(X, I))}(\mathbf{S}, \mathbf{T})=$ $\sum_{i \in I}\left(d\left(S_{i}, T_{i}\right)\right)<\infty$. This implies that $d\left(S_{i}, T_{i}\right) \rightarrow 0$ as $i \rightarrow \infty$. Thus by SCP we have that $d_{\mathrm{H}}\left(T_{i}(X), S_{i}(X)\right) \rightarrow 0$ as $i \rightarrow \infty$.

Let $x \in \operatorname{Acc}(\mathbf{S})$ and let us choose a sequence $\left\{x_{i}\right\}_{i \in I}$ such that $x_{i} \in S_{i}(X)$ for each $i \in I$. Note that $x \in \operatorname{Acc}(\mathbf{S})$ implies that there exists a subsequence $\left\{x_{i_{j}}\right\}_{j \in \mathbb{N}}$ such that $\lim _{i_{j} \rightarrow \infty} x_{i_{j}}=x$. Recall that $d\left(p, S_{i_{j}}(X)\right)=\inf _{y \in S_{i_{j}}(X)} d(y, x)<d\left(x_{i_{j}}, x\right)$ and hence $d\left(x, S_{i_{j}}(X)\right) \rightarrow 0$ as $i_{j} \rightarrow \infty$. We now have that

$$
d\left(x, T_{i_{j}}(X)\right) \leq d\left(x, S_{i_{j}}(X)\right)+d_{\mathrm{H}}\left(S_{i_{j}}(X), T_{i_{j}}(X)\right) .
$$

Thus $d\left(x, T_{i_{j}}(X)\right) \rightarrow 0$ as $i_{j} \rightarrow \infty$. Since $\mathbf{T}$ satisfies SSC, note that $x \in T_{i_{j}}(X)$ cannot hold for more than one $i_{j}$, which we denote by $i_{0}$. But then we have the following:

$$
x \in \overline{\bigcup_{i_{j} \neq i_{0}} T_{i_{j}}(X)} \backslash\left(\bigcup_{i_{j} \neq i_{0}} T_{i_{j}}(X)\right) .
$$

But now this implies that $x \in \operatorname{Acc}(\mathbf{T})$ and hence $\operatorname{Acc}(\mathbf{S}) \subseteq \operatorname{Acc}(\mathbf{T})$. Similarly one shows that $\operatorname{Acc}(\mathbf{T}) \subseteq \operatorname{Acc}(\mathbf{S})$. 
To state our next theorem we need the following definition.

Definition 8.4. Let $\mathbf{S} \in T((\mathrm{C}) \operatorname{IFS}(X, I))$ with $I$ infinite. For $r \in[0,1]$ define

$$
B(\mathbf{S}, r):=\left\{\mathbf{T} \in T((\mathrm{C}) \operatorname{IFS}(X, I)) \mid d_{T((\mathrm{C}) \operatorname{IFS}(X, I))}(\mathbf{S}, \mathbf{T})<r\right\}
$$

and

$$
\partial B(\mathbf{S}, r):=\left\{\mathbf{T} \in T((\mathrm{C}) \operatorname{IFS}(X, I)) \mid d_{T((\mathrm{C}) \operatorname{IFS}(X, I))}(\mathbf{S}, \mathbf{T})=r\right\} .
$$

Theorem 8.5. Let I be infinite. If $d_{T((C) I F S(X, I))}=\frac{d_{e x, T((C) I F S(X, I))}}{1+d_{e x, T((C) I F S(X, I))}}$ satisfies $S C P$, then we have that for $\mathbf{S} \in T((C) \operatorname{IFS}(X, I))$ the sets $B(\mathbf{S}, 1)$ and $\partial B(\mathbf{S}, 1)$ are open. In particular, this implies that:

(1) $T((C) \operatorname{IFS}(X, I))$ is disconnected if equipped with the metric $d_{T((C) I F S(X, I))}$.

(2) Let $\mathbf{T} \in T((C) \operatorname{IFS}(X, I))$ such that $\operatorname{Acc}(\mathbf{T}) \neq \operatorname{Acc}(\mathbf{S})$, then $\mathbf{T} \in \partial B(\mathbf{S}, 1)$.

(3) The map $\mathbf{T} \mapsto \operatorname{Acc}(\mathbf{T})$ is locally constant, i.e. is constant on $B(\mathbf{S}, 1)$.

(4) The map $\mathbf{T} \mapsto \operatorname{dim}_{\mathrm{H}} \operatorname{Acc}(\mathbf{T})$ is locally constant, i.e. is constant on $B(\mathbf{S}, 1)$.

(5) $T((C) I F S(X, I))$ has uncountably many connected components.

Proof. First observe that

$$
B(\mathbf{S}, 1)=\left\{\mathbf{T} \in T((\mathrm{C}) \operatorname{IFS}(X, I)) \mid d_{\mathrm{ex}, T(\operatorname{CIFS}(X, I))}(\mathbf{S}, \mathbf{T})<\infty\right\} .
$$

Let $\mathbf{Q} \in B(\mathbf{S}, 1)$. This means that $d_{\mathrm{ex}, T(\operatorname{CIFS}(X, I))}(\mathbf{S}, \mathbf{Q})<\infty$ and hence

$$
d_{\mathrm{ex}, T(\operatorname{CIFS}(X, I))}(\mathbf{S}, \mathbf{T})+d_{\mathrm{ex}, T(\operatorname{CIFS}(X, I))}(\mathbf{S}, \mathbf{Q})<\infty .
$$

Thus we have that

$$
\begin{aligned}
B(\mathbf{S}, 1)= & \left\{\mathbf{T} \in T((\mathrm{C}) \operatorname{IFS}(X, I)) \mid d_{\mathrm{ex}}, T(\operatorname{CIFS}(X, I))(\mathbf{S}, \mathbf{T})<\infty\right\} \\
= & \left\{\mathbf{T} \in T((\mathrm{C}) \operatorname{IFS}(X, I)) \mid d_{\mathrm{ex}}, T(\operatorname{CIFS}(X, I))\right. \\
& \left.+d_{\mathrm{ex}, T(\operatorname{CIFS}(X, I))}(\mathbf{S}, \mathbf{Q})<\infty\right\} \\
= & \left\{\mathbf{T} \in T((\mathrm{C}) \operatorname{IFS}(X, I)) \mid d_{\mathrm{ex}, T(\operatorname{CIFS}(X, I))}(\mathbf{Q}, \mathbf{T})<\infty\right\} \\
= & B(\mathbf{Q}, 1) .
\end{aligned}
$$

This implies that for any $\mathbf{Q} \in B(\mathbf{S}, 1)$ we have that $B(\mathbf{Q}, 1) \subseteq B(\mathbf{S}, 1)$ and hence $B(\mathbf{S}, 1)$ is open.

To see that $\partial B(\mathbf{S}, 1)$ is open, note that $d_{T((\mathrm{C}) \operatorname{IFS}(X, I))}(\mathbf{S}, \mathbf{T})=1$ means that $d_{\mathrm{ex}, T(\operatorname{CIFs}(X, I))}(\mathbf{S}, \mathbf{T})=\infty$. Let $\mathbf{Q} \in(\mathrm{C}) \operatorname{IFS}(X, I)$ with $d_{\mathrm{ex}, T(\operatorname{CIFS}(X, I))}(\mathbf{T}, \mathbf{Q})=\epsilon$ for some $\epsilon>0$ small enough which means that $d_{T((\mathrm{C}) \operatorname{IFS}(X, I))}(\mathbf{T}, \mathbf{Q})<\epsilon$. Thus we have that

$$
\begin{aligned}
d_{\mathrm{ex}, T(\operatorname{CIFs}(X, I))}(\mathbf{S}, \mathbf{T}) & \leq d_{\mathrm{ex}, T(\operatorname{CIFS}(X, I))}(\mathbf{S}, \mathbf{Q})+d_{\mathrm{ex}, T(\operatorname{CIFS}(X, I))}(\mathbf{Q}, \mathbf{T}) \\
& =d_{\mathrm{ex}, T(\operatorname{CIFS}(X, I))}(\mathbf{S}, \mathbf{Q})+\epsilon
\end{aligned}
$$

and hence $d_{T((\mathrm{C}) \operatorname{IFS}(X, I))}(\mathbf{S}, \mathbf{Q})=1$.

(1) This follows from the fact that the open sets $B(\mathbf{S}, 1)$ and $\partial B(\mathbf{S}, 1)$ are disjoint and $T((\mathrm{C}) \operatorname{IFS}(X, I))=B(\mathbf{S}, 1) \cup \partial B(\mathbf{S}, 1)$ if equipped with $d_{T((\mathrm{C}) \operatorname{IFS}(X, I))}$.

(2) It follows from Theorem 8.3 that $\mathbf{T} \in B(\mathbf{S}, 1)$ cannot hold if Acc $(\mathbf{T}) \neq$ $\operatorname{Acc}(\mathbf{S})$. Thus $\mathbf{T}$ is in $\partial B(\mathbf{S}, 1)$.

(3) and (4) The desired results follow immediately from Theorem 8.3 ,

(5) It was shown in [Hil11, Theorem 1] that for each $d \in \mathbb{N}$ and $\epsilon, j \in(0, d)$, there exists (C)IFS $\mathbf{S}$ acting on $\mathbb{R}^{d}$ such that

$$
\operatorname{dim}_{\mathrm{H}}\left(L_{\mathrm{J}}(\mathbf{S})\right)=j \quad \text { and } \quad \operatorname{dim}_{\mathrm{H}} L_{\mathrm{dyn}}(\mathbf{S}) \leq \epsilon .
$$


Now it follows from Theorem 8.2 that

$$
\operatorname{dim}_{\mathrm{H}} L_{\mathrm{J}}(\mathbf{S}) \leq \operatorname{dim}_{\mathrm{H}} \operatorname{Acc}(\mathbf{S}) \leq \max \left\{\operatorname{dim}_{\mathrm{H}} L_{\mathrm{dyn}}(\mathbf{S}), \operatorname{dim}_{\mathrm{H}} L_{\mathrm{J}}(\mathbf{S})\right\}
$$

Hence, in this case we have $\operatorname{dim}_{\mathrm{H}} L_{\mathrm{J}}(\mathbf{S})=\operatorname{dim}_{\mathrm{H}} \operatorname{Acc}(\mathbf{S})$. Therefore, $\operatorname{dim}_{\mathrm{H}} \operatorname{Acc}(\mathbf{S})$ (and thus $\operatorname{Acc}(\mathbf{S})$ ) can take uncountably many different values. Combining this with (1) and (3) gives the desired result.

\section{Continuity Revisited}

In this section we investigate which maps are continuous with respect to the different metrics introduced in this paper.

Theorem 9.1. If $T((C) \operatorname{IF} S(X, I))$ is equipped with a metric $d_{T((C) I F S(X, I))}=$ $\frac{d_{e x, T((C) I F S(X, I))}}{1+d_{e x, T((C) I F S(X, I))}}$ which satisfies $S C P$ and $\mathbb{R}^{d}$ is equipped with the topology induced by the Hausdorff distance, then we have

(1) $T((C) \operatorname{IFS}(X, I)) \rightarrow \mathbb{R}^{d}, \mathbf{S} \mapsto L(\mathbf{S})$ is continuous.

(2) $T((C) \operatorname{IFS}(X, I)) \rightarrow \mathbb{R}^{d}, \mathbf{S} \mapsto L_{d y n}(\mathbf{S})$ is continuous.

(3) $T((C) \operatorname{IFS}(X, I)) \rightarrow \mathbb{R}^{d}, \mathbf{S} \mapsto L_{J}(\mathbf{S})$ is not continuous in general.

Proof. (1) Let $\mathbf{S}, \mathbf{T} \in T((\mathrm{C}) \operatorname{IFS}(X, I))$. First note that for any $\epsilon>0$ there exist $k, m \in \mathbb{N}$ such that

$$
\operatorname{diam}\left(S_{\mathbf{i}}(X)\right)<\frac{\epsilon}{3} \quad \text { for all } \quad \mathbf{i} \in I^{k}
$$

Let $n=\max \{k, m\}$ and observe that

$$
\begin{aligned}
d_{\mathrm{H}}(L(\mathbf{S}), L(\mathbf{T})) \leq d_{\mathrm{H}} & \left(L(\mathbf{S}), \bigcup_{\mathbf{i} \in I^{n}} S_{\mathbf{i}}(X)\right) \\
& +d_{\mathrm{H}}\left(\bigcup_{\mathbf{i} \in I^{n}} S_{\mathbf{i}}(X), \bigcup_{\mathbf{i} \in I^{n}} T_{\mathbf{i}}(X)\right)+d_{\mathrm{H}}\left(\bigcup_{\mathbf{i} \in I^{n}} T_{\mathbf{i}}(X), L(\mathbf{T})\right),
\end{aligned}
$$

where $d_{\mathrm{H}}$ denotes the Hausdorff distance.

Since for all $\mathbf{i} \in I^{n}$ we have that $\operatorname{diam}\left(S_{\mathbf{i}}(X)\right)<\frac{\epsilon}{3}$ and $S_{\mathbf{i}}(X) \cap L(\mathbf{S}) \neq \emptyset$, then $\bigcup_{\mathbf{i} \in I^{n}} S_{\mathbf{i}}(X)$ is contained in the $\frac{\epsilon}{3}$-neighborhood of $L(\mathbf{S})$. In order to see that $L(\mathbf{S})$ is contained in the $\frac{\epsilon}{3}$-neighborhood of $\bigcup_{\mathbf{i} \in I^{n}} S_{\mathbf{i}}(X)$ it is sufficient to recall that $L(\mathbf{S}) \subset \overline{\bigcup_{\mathbf{i} \in I^{n}} S_{\mathbf{i}}(X)}$. This shows that $d_{\mathrm{H}}\left(L(\mathbf{S}), \bigcup_{\mathbf{i} \in I^{n}} S_{\mathbf{i}}(X)\right)<\frac{\epsilon}{3}$.

A similar argument shows that $d_{\mathrm{H}}\left(L(\mathbf{T}), \bigcup_{\mathbf{i} \in I^{n}} T_{\mathbf{i}}(X)\right)<\frac{\epsilon}{3}$.

For the middle term $d_{\mathrm{H}}\left(\bigcup_{\mathbf{i} \in I^{n}} S_{\mathbf{i}}(X), \bigcup_{\mathbf{i} \in I^{n}} T_{\mathbf{i}}(X)\right)$ we argue as follows. First, we show that for each $i \in I$ the map $\mathbf{S} \mapsto S_{i}(X)$ is continuous. This can be seen 
from the following inequality

$$
\begin{aligned}
d_{\mathrm{H}}\left(S_{i}(X), T_{i}(X)\right) & =\max \left\{\sup _{x \in S_{i}(X)} \inf _{y \in T_{i}(X)} d(x, y), \sup _{y \in T_{i}(X)} \inf _{x \in S_{i}(X)} d(x, y)\right\} \\
& =\max \left\{\sup _{a \in X} \inf _{b \in X} d\left(S_{i}(a), T_{i}(b)\right), \quad \sup _{b \in X} \inf _{a \in X} d\left(S_{i}(a), T_{i}(b)\right)\right\} \\
& \leq \max \left\{\sup _{a \in X} d\left(S_{i}(a), T_{i}(a)\right), \quad \sup _{b \in X} d\left(S_{i}(b), T_{i}(b)\right)\right\} \\
& =\left\|S_{i}-T_{i}\right\|_{X} \\
& \leq d_{\mathrm{ex}, T((\mathrm{C}) \operatorname{IFS}(X, I))}(\mathbf{S}, \mathbf{T}) .
\end{aligned}
$$

If $\mathbf{i} \in I^{n}$, then this calculation changes as follows. For ease of exposition, let us only consider the term $\sup _{x \in S_{\mathbf{i}}(X)} \inf _{y \in T_{\mathbf{i}}(X)} d(x, y)$,

$$
\sup _{x \in S_{\mathbf{i}}(X)} \inf _{y \in T_{\mathbf{i}}(X)} d(x, y)=\sup _{a \in X} \inf _{b \in X} d\left(S_{\mathbf{i}}(a), T_{\mathbf{i}}(b)\right) \leq \sup _{a \in X} d\left(S_{\mathbf{i}}(a), T_{\mathbf{i}}(a)\right) .
$$

Now note that for all $a \in X$ we have that

$$
\begin{aligned}
d\left(S_{i_{1} i_{2}}(a), T_{i_{1} i_{2}}(a)\right) & =d\left(S_{i_{2}} \circ S_{i_{1}}(a), T_{i_{2}} \circ T_{i_{1}}(a)\right) \\
& \leq d\left(S_{i_{2}}\left(S_{i_{1}}(a)\right), S_{i_{2}}\left(T_{i_{1}}(a)\right)\right)+d\left(S_{i_{2}}\left(T_{i_{1}}(a)\right), T_{i_{2}}\left(T_{i_{1}}(a)\right)\right) \\
& \leq d\left(S_{i_{1}}(a), T_{i_{1}}(a)\right)+d\left(S_{i_{2}}(b), T_{i_{2}}(b)\right) \\
& \leq\left\|S_{i_{1}}-T_{i_{1}}\right\|_{X}+\left\|S_{i_{2}}-T_{i_{2}}\right\|_{T_{i_{1}}(X)} \\
& \leq 2 \cdot \max _{i \in I}\left\|S_{i}-T_{i}\right\|_{X} \\
& \leq 2 \cdot d_{\text {ex }}, T((\mathrm{C}) \operatorname{IFS}(X, I))
\end{aligned}
$$

where in (9.3) we have used that $S_{i_{2}}$ is a contraction, i.e. $d\left(S_{i_{2}}(x), S_{i_{2}}(y)\right)<d(x, y)$ and that $b:=T_{i_{1}}(a)$.

Thus, for $\mathbf{i} \in I^{n}$ and for all $a \in X$ we have that

$$
d\left(S_{\mathbf{i}}(a), T_{\mathbf{i}}(a)\right) \leq n \cdot \max _{i \in I}\left\|S_{i}-T_{i}\right\|_{X} \leq n \cdot d_{\mathrm{ex}, T((\mathrm{C}) \operatorname{IFS}(X, I))}(\mathbf{S}, \mathbf{T}) .
$$

Hence, if $d_{T((\mathrm{C}) \operatorname{IFS}(X, I))}(\mathbf{S}, \mathbf{T})<\delta:=\frac{\frac{\epsilon}{3 n}}{1+\frac{\epsilon}{3 n}}$, then we have that

$$
\frac{d_{\mathrm{ex}, T((\mathrm{C}) \operatorname{IFS}(X, I))}(\mathbf{S}, \mathbf{T})}{1+d_{\mathrm{ex}, T((\mathrm{C}) \operatorname{IFS}(X, I))}(\mathbf{S}, \mathbf{T})}<\frac{\frac{\epsilon}{3 n}}{1+\frac{\epsilon}{3 n}}
$$

if and only if

$$
\begin{aligned}
d_{\mathrm{ex}, T((\mathrm{C}) \operatorname{IFS}(X, I))}(\mathbf{S}, \mathbf{T})+\frac{\epsilon}{3 n} \cdot d_{\mathrm{ex}, T((\mathrm{C}) \operatorname{IFS}(X, I))}(\mathbf{S}, \mathbf{T}) & \\
& <\frac{\epsilon}{3 n}+\frac{\epsilon}{3 n} \cdot d_{\mathrm{ex}, T((\mathrm{C}) \operatorname{IFS}(X, I))}(\mathbf{S},
\end{aligned}
$$

if and only if

$$
d_{\mathrm{ex}, T((\mathrm{C}) \operatorname{IFS}(X, I))}(\mathbf{S}, \mathbf{T})<\frac{\epsilon}{3 n} .
$$

Therefore $\sup _{a \in X} d\left(S_{\mathbf{i}}(a), T_{\mathbf{i}}(a)\right)<\frac{\epsilon}{3}$. This implies that $d_{\mathrm{H}}\left(S_{\mathbf{i}}(X), T_{\mathbf{i}}(X)\right)<\frac{\epsilon}{3}$ and hence $d_{\mathrm{H}}\left(\bigcup_{\mathbf{i} \in I^{n}} S_{\mathbf{i}}(X), \bigcup_{\mathbf{i} \in I^{n}} T_{\mathbf{i}}(X)\right) \leq \max _{\mathbf{i} \in I^{n}} d_{\mathrm{H}}\left(S_{\mathbf{i}}(X), T_{\mathbf{i}}(X)\right)<\frac{\epsilon}{3}$.

Combining these observations we have shown that if $d_{T((\mathrm{C}) \operatorname{IFS}(X, I))}(\mathbf{S}, \mathbf{T})<\delta:=$ $\frac{\epsilon}{3 n}$, then $d_{\mathrm{H}}(L(\mathbf{S}), L(\mathbf{T}))<\epsilon$, which completes the proof. 
(2) A similar argument works for $L_{\mathrm{dyn}}(\mathbf{S})$ instead of $L(\mathbf{S})$. However, since $L_{\mathrm{dyn}}(\mathbf{S})$ is in general not closed, we need to use the topology induced by the Hausdorff distance instead of the Hausdorff distance.

(3) Let $X$ be the unit square, i.e. $X:=[0,1] \times[0,1] \subset \mathbb{R}^{2}$. Further let $\mathbf{S}_{0}:=$ $\left\{S_{j}\right\}_{j \in\{1, \ldots, 5\}}$ be given by $S_{j}(x, y)=\frac{1}{3}(x, y)+a_{j}$ with $a_{1}=(0,0), a_{2}=(0,2 / 3)$, $a_{3}=(2 / 3,0), a_{4}=(2 / 3,2 / 3)$ and $S_{5}(x)=\frac{1}{4}(x, y)+\left(\frac{3}{8}, \frac{3}{8}\right)$. We will now inductively add new generators to create an infinite IFS $\mathbf{S}$ as follows. Let $C_{i}(x)=\frac{x}{3}+i \frac{2}{3}$ for $i \in I=\{0,1\}$. Now let $F_{\mathbf{i}}(x, y)=\left(\frac{1}{4} C_{\mathbf{i}}(x), \frac{1}{4^{n}} y\right)+\left(\frac{3}{8}, \frac{5}{8}\right)$ for $\mathbf{i} \in I^{n}$. We define an IFS $\mathbf{S}_{n}$ with $2^{n}+5$ generators by $\mathbf{S}_{n}:=\mathbf{S}_{0} \cup \bigcup_{\mathbf{i} \in I^{n}}\left\{F_{\mathbf{i}}\right\}$. Note that $\mathbf{S}_{n}$ satisfies SSC. We define $\mathbf{S}:=\bigcup_{n \in \mathbb{N}} \mathbf{S}_{n}$ (see Figure 9.0.1). Observe that by construction the set of accumulation points $\operatorname{Acc}(\mathbf{S})$ is exactly given by the set of the limit points of the finite IFS $\mathbf{S}_{0}$ intersected with $y$-coordinate $\frac{5}{8}$. Therefore, we have that $\operatorname{Acc}(\mathbf{S}) \subset L_{\text {dyn }}(\mathbf{S})$. This implies that $L_{\mathrm{J}}(\mathbf{S})=\emptyset$. Also, note that $\operatorname{Acc}(\mathbf{S})$ is a scaled down copy of the middle third Cantor set. Now for $\epsilon>0$, let $\mathbf{S}_{\epsilon}$ be an IFS obtained from $\mathbf{S}$ by scaling down the generators of $\mathbf{S}_{0}$ by $1-\epsilon$. For these $\mathbf{S}_{\epsilon}$ we now have that $\operatorname{Acc}\left(\mathbf{S}_{\epsilon}\right) \cap L_{\text {dyn }}\left(\mathbf{S}_{\epsilon}\right)=\emptyset$ and hence $L_{\mathrm{J}}\left(\mathbf{S}_{\epsilon}\right)=\mathcal{O}_{\mathbf{S}_{\epsilon}}\left(\operatorname{Acc}\left(\mathbf{S}_{\epsilon}\right)\right) \neq \emptyset$. Finally, note that for every $\delta>0$ we can find sufficiently small $\epsilon>0$ such that $d\left(\mathbf{S}, \mathbf{S}_{\epsilon}\right)<\delta$. However, $L_{\mathrm{J}}\left(\mathbf{S}_{\epsilon}\right)$ is not contained in any $\delta$-neighborhood of $L_{\mathrm{J}}(\mathbf{S})=\emptyset$ and hence the Hausdorff distance between $L_{\mathrm{J}}\left(\mathbf{S}_{\epsilon}\right)$ and $L_{\mathrm{J}}(\mathbf{S})$ is not finite. This implies that the map $\mathbf{T} \mapsto L_{\mathrm{J}}(\mathbf{T})$ is not continuous at $\mathbf{S}$.

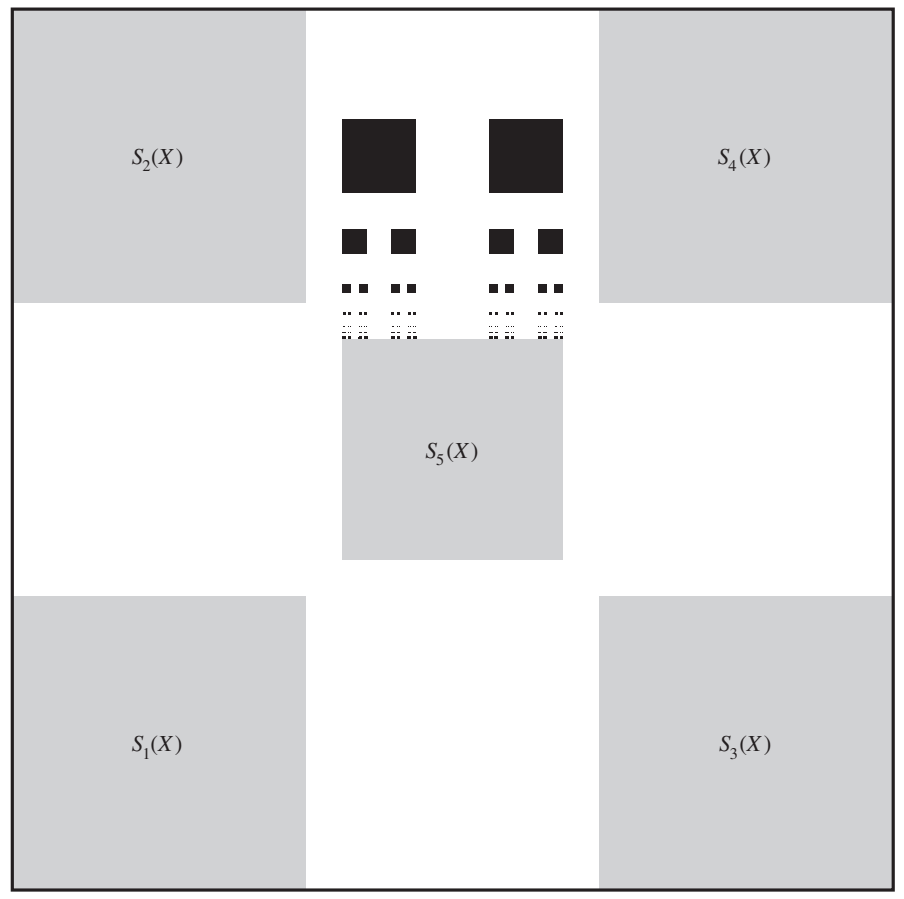

Figure 9.0.1. IFS $\mathbf{S}$ in the proof of Theorem 9.1 (Part 3). 
Theorem 9.2. Let $T((C) \operatorname{IF} S(X, I))$ be equipped with a metric $d_{T((C) I F S(X, I))}=$

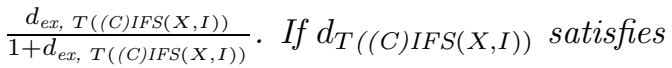
(1) $L C P$,
then $T((C) I F S(X, I)) \rightarrow \mathbb{R}, \mathbf{S} \mapsto \operatorname{dim}_{\mathrm{H}} L_{d y n}(\mathbf{S})$ is con-
(2) $S C P$ and LCP, then $T((C) \operatorname{IFS}(X, I)) \rightarrow \mathbb{R}, \mathbf{S} \mapsto \operatorname{dim}_{\mathrm{H}} L(\mathbf{S})$ is con- tinuous.
(3) SCP, $T((C) \operatorname{IFS}(X, I))$
continuous in general. tinuous.

Proof. (1) It was shown in RU05 that the Hausdorff dimension function $h$ : $T((\mathrm{C}) \operatorname{IFS}(X, I)) \cup \partial_{\mathrm{ess}}^{\mathrm{osc}} T((\mathrm{C}) \operatorname{IFS}(X, I)) \rightarrow[0, \infty), \mathbf{S} \mapsto \operatorname{dim}_{\mathrm{H}} L_{\mathrm{dyn}}(\mathbf{S})$, is continuous when $T((\mathrm{C}) \operatorname{IFS}(X, I)) \cup \partial_{\mathrm{ess}}^{\mathrm{osc}} T((\mathrm{C}) \operatorname{IFS}(X, I))$ is equipped with the $\lambda$-topology. The desired result now follows immediately from the fact that the metric $d_{T((\mathrm{C}) \operatorname{IFS}(X, I))}$ satisfies LCP.

(2) It was shown in [Hil11, Corollary 2] that $\operatorname{dim}_{\mathrm{H}} L(\mathbf{S})=\max \left\{\operatorname{dim}_{\mathrm{H}} \operatorname{Acc}(\mathbf{S})\right.$, $\left.\operatorname{dim}_{\mathrm{H}} L_{\mathrm{dyn}}(\mathbf{S})\right\}$. Combining this with Theorem 8.5 (Part (4) and Part (1)) above gives the required result.

(3) Consider IFSs $\mathbf{S}$ and $\mathbf{S}_{\epsilon}$ as in the proof of Theorem 9.1 (Part (3)). Then the map $\mathbf{T} \mapsto \operatorname{dim}_{\mathrm{H}} L_{\mathrm{J}}(\mathbf{T})$ is not continuous at $\mathbf{S}$, since for the aforementioned $\mathbf{S}_{\epsilon}$ we have that $\operatorname{dim}_{\mathrm{H}} L_{\mathrm{J}}\left(\mathbf{S}_{\epsilon}\right)=\frac{\log 2}{\log 3}$, while for $\mathbf{S}$ we have that $\operatorname{dim}_{\mathrm{H}} L_{\mathrm{J}}(\mathbf{S})=0$.

Let Discont $\operatorname{dim}_{\mathrm{H}} L_{\mathrm{J}}:=\left\{\mathbf{S} \in T((\mathrm{C}) \operatorname{IFS}(X, I)) \mid \mathbf{T} \mapsto \operatorname{dim}_{\mathrm{H}} L_{\mathrm{J}}(\mathbf{T})\right.$ is not continuous at $\mathbf{S}\}$ be the set of points of discontinuity of the function $\mathbf{T} \mapsto \operatorname{dim}_{\mathrm{H}} L_{\mathrm{J}}(\mathbf{T})$. We then have the following observations.

Theorem 9.3. Let $T((C) \operatorname{IFS}(X, I))$ be equipped with a metric $d_{T((C) I F S(X, I))}$ which satisfies SCP.

(1) Additionally, assume that $d_{T((C) I F S(X, I))}$ satisfies LCP. Then we have that the map: $T((C) \operatorname{IFS}(X, I)) \rightarrow \mathbb{R}, \mathbf{S} \mapsto \operatorname{dim}_{\mathrm{H}} L_{J}(\mathbf{S})$ is continuous on $\left\{\mathbf{T} \in T((C) I F S(X, I)) \mid \operatorname{dim}_{\mathrm{H}} L_{d y n}(\mathbf{T})<\operatorname{dim}_{\mathrm{H}} L_{J}(\mathbf{T})\right\}$.

(2) Assume that the phase space $X \subset \mathbb{R}$ is a union of finitely many intervals. Then the map: $T((C) \operatorname{IFS}(X, I)) \rightarrow \mathbb{R}, \mathbf{S} \mapsto \operatorname{dim}_{\mathrm{H}} L_{J}(\mathbf{S})$ is locally constant and hence Discont dim $_{\mathrm{H}} L_{J}=\emptyset$.

(3) Assume that the phase space $X$ is star-shaped with center c. If $\mathbf{S} \in$ Discont $_{\operatorname{dim}_{\mathrm{H}} L_{J}}$, then $\mathbf{S}$ is the limit of a sequence of $(C)$ IFS $\left\{\mathbf{S}_{k}\right\}_{k \in \mathbb{N}}$ which satisfies

(a) $\mathcal{O}_{\mathbf{S}_{k}} \operatorname{Acc}\left(\mathbf{S}_{k}\right)=L_{J}\left(\mathbf{S}_{k}\right)$,

(b) $\mathbf{S}_{k} \notin$ Discont $_{\mathrm{dim}_{\mathrm{H}} L_{J}}$, i.e. $\mathbf{S}_{k}$ is a point of continuity of the function $\mathbf{S} \mapsto \operatorname{dim}_{\mathrm{H}} L_{J}(\mathbf{S})$.

Proof. (1) Note that if $\mathbf{T}$ satisfies $\operatorname{dim}_{\mathrm{H}} L_{\mathrm{dyn}}(\mathbf{T})<\operatorname{dim}_{\mathrm{H}} L_{\mathrm{J}}(\mathbf{T})$ (which is equivalent to $\left.\operatorname{dim}_{\mathrm{H}} L_{\mathrm{dyn}}(\mathbf{T})<\operatorname{dim}_{\mathrm{H}} \operatorname{Acc}(\mathbf{T})\right)$, then $\operatorname{dim}_{\mathrm{H}} L(\mathbf{T})=\operatorname{dim}_{\mathrm{H}} \operatorname{Acc}(\mathbf{T})=\operatorname{dim}_{\mathrm{H}} L_{\mathrm{J}}(\mathbf{T})$. By Theorem 9.2 we have that the maps $\mathbf{S} \mapsto \operatorname{dim}_{\mathrm{H}} L(\mathbf{S})$ and $\mathbf{S} \mapsto \operatorname{dim}_{\mathrm{H}} L_{\mathrm{dyn}}(\mathbf{S})$ are continuous. Moreover, by Theorem 8.5 the map $\mathbf{S} \mapsto \operatorname{dim}_{\mathrm{H}} \operatorname{Acc}(\mathbf{S})$ is locally constant. Combining these facts we have that for a given (C)IFS $\mathbf{T}$ with $\operatorname{dim}_{\mathrm{H}} L_{\mathrm{dyn}}(\mathbf{T})<\operatorname{dim}_{\mathrm{H}} L_{\mathrm{J}}(\mathbf{T})$ there exists a sufficiently small neighborhood $U$ of $\mathbf{T}$ such that

$$
\operatorname{dim}_{\mathrm{H}} L_{\mathrm{dyn}}(\mathbf{S})<\operatorname{dim}_{\mathrm{H}} L(\mathbf{S})=\operatorname{dim}_{\mathrm{H}} \operatorname{Acc}(\mathbf{S})=\operatorname{dim}_{\mathrm{H}} L_{\mathrm{J}}(\mathbf{S})=\operatorname{dim}_{\mathrm{H}} \operatorname{Acc}(\mathbf{T})
$$

for all $\mathbf{S} \in U$. This implies that the map $\mathbf{S} \mapsto \operatorname{dim}_{\mathrm{H}} L_{\mathrm{J}}(\mathbf{S})$ is continuous on $U$. 
(2) It was shown in [Hil11, Theorem 7] that if $X \subset \mathbb{R}$ is a union of finitely many intervals, then $\operatorname{dim}_{\mathrm{H}} L_{\mathrm{J}}(\mathbf{S})=\operatorname{dim}_{\mathrm{H}} \operatorname{Acc}(\mathbf{S})$. Combining this with the fact that the map $\mathbf{S} \mapsto \operatorname{Acc}(\mathbf{S})$ is locally constant the required result follows.

(3) Let $\mathbf{S}=\left\{S_{i}\right\}_{i \in I} \in$ Discont $_{\operatorname{dim}_{\mathrm{H}} L_{\mathrm{J}}}$ and for $k \in \mathbb{N}$ define $\mathbf{S}_{k}=\left\{S_{i, k}\right\}_{i \in I}$ by letting

$$
S_{i, k}(x):=S_{i}\left(\left(\frac{1}{k} \cdot 2^{-i}\right) x+\left(1-\frac{1}{k} \cdot 2^{-i}\right) c\right) .
$$

(a) Now observe that by construction $\mathbf{S}_{k}$ satisfies the Nestedness Condition (NC) introduced in [Hil09]. Recall that an IFS $\mathbf{S}$ satisfies the Nestedness Condition if there exists an open set $U^{\prime}$ such that for all $i \in I$ we have $S_{i}(X) \subset U^{\prime}$ and $\overline{U^{\prime}} \subset$ Int $X$. Hence, we have that $L_{\text {dyn }}\left(\mathbf{S}_{k}\right) \subset \bigcup_{i \in I} \int S_{i, k}(X)$. Therefore, it follows from [Hil11, Lemma 9] that $\mathcal{O}_{\mathbf{S}_{k}}\left(\operatorname{Acc}\left(\mathbf{S}_{k}\right)\right)=L_{\mathrm{J}}\left(\mathbf{S}_{k}\right)$.

(b) Combining the latter fact with the continuity of the map $\mathbf{T} \mapsto L_{\mathrm{dyn}}(\mathbf{T})$ we have that there exists a small neighborhood $U$ around each $\mathbf{S}_{k}$ such that $\mathcal{O}_{\mathbf{T}} \operatorname{Acc}(\mathbf{T})=L_{\mathrm{J}}(\mathbf{T})$ for all $\mathbf{T} \in U$. Hence, $\operatorname{dim}_{\mathrm{H}} L_{\mathrm{J}}(\mathbf{T})=\operatorname{dim}_{\mathrm{H}} \operatorname{Acc}(\mathbf{T})$ for all $\mathbf{T} \in U$ which implies that $\mathbf{T} \mapsto \operatorname{dim}_{\mathrm{H}} L_{\mathrm{J}}(\mathbf{T})$ is locally constant. Therefore, $\mathbf{S}_{k} \notin$ Discont $_{\operatorname{dim}_{\mathrm{H}} L_{\mathrm{J}}}$.

We conclude the paper by remarking that the metrics we introduced are very strong in the following sense. Most of the maps we investigated are continuous with respect to our metrics. The maps which were shown to be discontinuous in general can only be made continuous with respect to the discrete metric. This indicates that our metrics are good for studying deformations of (C)IFS.

\section{REFERENCES}

[Bar89] M. F. Barnsley, Lecture notes on iterated function systems, Chaos and Fractals (Providence, RI, 1988), 127-144, Proc. Sympos. Appl. Math., 39, Amer. Math. Soc., Providence, RI, 1989. MR1010239

[Bar93] _ Fractals everywhere, 2nd ed., Academic Press, Boston, 1993. MR.1231795 (94h:58101)

[Bar06] , Superfractals, Cambridge University Press, Cambridge, 2006. MR2254477 (2008c:28006)

[BBG] T. Bedford, S. Borodachov, and J. S. Geronimo, A topological separation condition for fractal attractors, arXiv:0911.2126.

[BD85] M. F. Barnsley and S. Demko, Iterated function systems and the global construction of fractals, Proc. Roy. Soc. London Ser. A 399 (1985), 243-275. MR799111 (87c:58051)

[BGH85] M.F. Barnsley, J. S. Geronimo, and A. N. Harrington, Condensed Julia sets, with application to a fractal lattice model Hamiltonian, Transactions of the AMS 288 (1985), 537-561. MR776392 (86h:58088)

[BP92] R. Bennedetti and C. Petronio, Lectures on hyperbolic geometry, Springer-Verlag, Berlin, 1992. MR:1219310 (94e:57015)

[Hil09] M. Hille, Resonances for graph directed Markov systems, and geometry of infinitely generated dynamical systems, University of St. Andrews, 2009.

[Hil11] _ Remarks on limit sets of infinite iterated function systems, Monatsh. Math., DOI 10.1007/s00605-011-0357-6 (2011).

[HSa] M. Hille and N. Snigireva, Teichmüller space for affine iterated function systems, in preparation.

[HSb] Teichmüller space for graph directed Markov systems, in preparation.

[Hut81] J. E. Hutchinson, Fractals and self-similarity, Indiana Univ. Math. J. 30 (1981), no. 5, 713-747. MR625600 (82h:49026)

[KS08] M. Kesseböhmer and B. O. Stratmann, Refined measurable rigidity and flexibility for conformal iterated function systems, New York J. Math. 14 (2008), 33-51. MR.2383585 (2009i:37060) 
[MS98] C. T. McMullen and D. P. Sullivan, Quasiconformal homeomorphisms and dynamics. III. The Teichmüller space of a holomorphic dynamical system, Adv. Math. 135 (1998), 351-395. MR1620850 (99e:58145)

[MU96] D. Mauldin and M. Urbanski, Dimensions and measures in infinite iterated function systems, Proc. London Math. Soc. 73 (1996), 105-154. MR1387085 (97c:28020)

[OS07] L. Olsen and N. Snigireva, $L^{q}$ spectra and Rényi dimensions of in-homogeneous selfsimilar measures, Nonlinearity 20 (2007), 151-175. MR2285110(2009a:28024)

[OS08a] _ In-homogenous self-similar measures and their Fourier transforms, Math. Proc. Camb. Phil. Soc. 144 (2008), 465-493. MR2405903 (2009i:42004)

[OS08b] _ Multifractal spectra of in-homogenous self-similar measures, Indiana U. Math. J. 57 (2008), 1789-1844. MR2440882 (2009k:37050)

[PRF08] A. A. Pinto, D. A. Rand, and F. Ferreira, Fine structures of hyperbolic diffeomorphisms, Springer-Verlag, 2008. MR2464147 (2010e:37036)

[RSU09] M. Roy, H. Sumi, and M. Urbanski, $\lambda$ topology vs. pointwise topology, Ergodic Theory Dynam. Systems 29 (2009), 685-713. MR2486790 (2010c:37050)

[RU05] M. Roy and M. Urbanski, Regularity properties of Hausdorff dimension in infinite conformal iterated function systems, Ergodic Theory Dynam. Systems 25 (2005), 19611983. MR2183304 (2008c:37042)

Humboldt-Universität zu Berlin, Institut für Mathematik, Unter den Linden 6, D10099 Berlin, Germany

E-mail address: hille@math.hu-berlin.de

Mathematical Sciences Institute, John Dedman Building 27, The Australian National University, Canberra ACT 0200, Australia

E-mail address: Nina.Snigireva@anu.edu.au 